\title{
A Simple Finite Element Formulation for Computing Stress Singularities at Bimaterial Interfaces
}

\author{
K.Y.Sze and Hai-tao Wang \\ Department of Mechanical Engineering, The University of Hong Kong \\ Pokfulam Road, Hong Kong SAR, P.R.CHINA
}

\begin{abstract}
By using the weak form of the governing equations for sectorial bimaterial domains and assuming that the displacement field is proportional to the $(\lambda+1)$-th power of the distance from the singular stress point, a second order characteristic matrix equation on $\lambda$ is derived by a one-dimensional finite element formulation that only discretizes the domain circumferentially. Numerical examples covering a variety of interfacial singularities are presented to demonstrate the efficacy of the formulation. Accurate solutions are yielded by very few elements whereas convergence can be attained by either h- or p-refinement. The related procedures are programmed in a short MAPLE worksheet given in the appendix.
\end{abstract}

keyword : finite element, stress singularities, interface, crack, free edge, bimaterial 


\section{INTRODUCTION}

Co-usage of different materials in modern engineering devices and components has been very common as a result of various functional requirements on mechanical, thermal and electric properties. The mismatch of the material properties give rise to singular stresses at geometric discontinuities along bimaterial interfaces as shown in Fig.1. The design and operation of these devices and components inevitably require a understanding of the singularity stresses which are often assumed to have the asymptotic form : $\sigma \propto r^{\lambda} G(\theta)$ where $(r, \theta)$ are the polar coordinates originated at the singular stress point, $\lambda$ is the singularity order and $G$ gives the angular variation. $\operatorname{Re}(\lambda)$ is larger than -1 and smaller than zero. Otherwise, the strain energy will be unbounded and the stress will be non-singular, respectively. $\lambda$ and $G(\theta)$ are referred to as eigenpair. While the proportionality constant or the intensity factor depends on the overall dimension and the external loading of the bimaterial system, the eigenpair is intrinsic to the material properties and the geometric configuration at the singular stress point.

Like most engineering problems, derivation of analytical eigenpair solutions is either limited to very simple configurations or requiring high level of mathematical competence. Naturally, numerical methods for eigenpair determination have been resorted to. Some of the previous works are here briefly reviewed. For the asymptotic stresses, the pertinent asymptotic displacements relative to the singular point will be of the form : $u \propto r^{\lambda+1} F(\theta)$. Yamada \& Okumura [1] employed a singular transformation technique with which the displacement of the devised sectorial elements become proportional to $r^{\lambda+1}$. By modelling the crack tip region with the sectorial elements and making use of the virtual work principle, a quadratic characteristic matrix equation on $\lambda$ can be set up. The same approach was later employed by Pageau and his coworkers for bimaterial systems making up of anisotropic materials [2,3]. On the other hand, Barsoum [4,5] proposed the finite element iterative method which can be used as a substructuring method to extract both the intensity and the eigenpair. The iterative procedure for determining $\lambda$ was devised based on a variant of the relation $u\left(R_{o}, \theta\right) / u\left(R_{i}, \theta\right)=\left(R_{o} / R_{i}\right)^{\lambda+1}$. Barsoum suggested that the ratio of $R_{o}$ to $R_{i}$ should be larger than 100. Yosibash \& Szabo [6] proposed the Steklov and modified Steklov methods which again assume the same asymptotic form of the displacement. The methods start with a weak form of the homogeneous equilibrium equations and result in a linear characteristic matrix equation. $\mathrm{Gu} \&$ Belytschko [7] proposed several methods to predict the asymptotic stress field. By using a spectral overlay FE method which is essentially a global-local FE approach, the FE approximation around the singular stress point is enhanced with higher order displacement modes. To characterize the singularity, a least square procedure is employed over a small sub-domain surrounding the singular 
point for fitting a stress field of the form $\sigma=A r^{\lambda}+B r+C$ to the FE solution. The procedure appears to be rather sensitive to the chosen size of the sub-domain and is not applicable to complex $\lambda$ 's which lead to highly oscillatory stress at the crack tip vicinity, see reference [8] for discussion. $\mathrm{Gu} \&$ Belytschko also derived a stress function-based and a displacement-based methods which result respectively in fourth and second order characteristic matrix equations [7].

In this paper, a simple FE numerical method for eigenpair determination is devised based on the weak form of the governing equations. A one-dimensional finite element formulation is employed that only discretizes the domain and the asymptotic displacement circumferentially. A number of benchmark problems are studied and the accuracy yielded by the present method is satisfactory.

\section{DIVERGENCE THEOREM FOR 2/D SECTORIAL DOMAIN}

Divergence theorem is a fundamental relation in variational methods that relates the equilibrium relation, strain energy and the work done by the surface force. The version expressed with respect to polar coordinates for a sectorial domain is presented in the section. Under the coordinate system, the strain-displacement relation is :

$$
\boldsymbol{\epsilon}=\left\{\varepsilon_{r}, \varepsilon_{\theta}, \gamma_{r \theta}\right\}^{T}=\left\{\frac{\partial u}{\partial r}, \frac{u}{r}+\frac{1}{r} \frac{\partial v}{\partial \theta}, \frac{1}{r} \frac{\partial u}{\partial \theta}+r \frac{\partial}{\partial r}\left(\frac{v}{r}\right)\right\}^{T}
$$

in which $u$ and $v$ are the radial and circumferential displacements whereas $\varepsilon_{r}, \varepsilon_{\theta}$ and $\gamma_{r \theta}$ are the strain components. On the other hand, the homogeneous stress equilibrium conditions are :

$$
\frac{\partial\left(r \sigma_{r}\right)}{\partial r}+\frac{\partial \tau_{r \theta}}{\partial \theta}-\sigma_{\theta}=0, \frac{\partial \sigma_{\theta}}{\partial \theta}+\frac{\partial\left(r \tau_{r \theta}\right)}{\partial r}+\tau_{r \theta}=0
$$

where $\sigma_{r}, \sigma_{\theta}$ and $\tau_{r \theta}$ are the stress components. It is a straight forward matter to prove the following identity :

$$
\frac{1}{r} \delta \mathbf{u}^{T}\left\{\begin{array}{l}
\frac{\partial\left(r \sigma_{r}\right)}{\partial r}+\frac{\partial \tau_{r \theta}}{\partial \theta}-\sigma_{\theta} \\
\frac{\partial \sigma_{\theta}}{\partial \theta}+\frac{\partial\left(r \tau_{r \theta}\right)}{\partial r}+\tau_{r \theta}
\end{array}\right\}+\delta \boldsymbol{\epsilon}^{T} \boldsymbol{\sigma}=\frac{1}{r}\left[\frac{\partial}{\partial r}\left(r \delta \mathbf{u}^{T}\left\{\begin{array}{c}
\sigma_{r} \\
\tau_{r \theta}
\end{array}\right\}\right)+\frac{\partial}{\partial \theta}\left(\delta \mathbf{u}^{T}\left\{\begin{array}{c}
\tau_{r \theta} \\
\sigma_{\theta}
\end{array}\right\}\right)\right]
$$

in which $\delta$ is the variational operator, $\mathbf{u}=\{u, v\}^{T}$ and $\boldsymbol{\sigma}=\left\{\sigma_{r}, \sigma_{\theta}, \tau_{r \theta}\right\}^{T}$ are the vectors of displacement and stress components. By integrating the above equation over the two-dimensional sectorial domain $\Omega$ bounded by $\theta=\theta_{s}, \theta=\theta_{e}$ and $r=R$ as shown in Fig.2a, we have 


$$
\int_{\Omega}\left(\frac{1}{r} \delta \mathbf{u}^{T}\left\{\begin{array}{l}
\frac{\partial\left(r \sigma_{r}\right)}{\partial r}+\frac{\partial \tau_{r \theta}}{\partial \theta}-\sigma_{\theta} \\
\frac{\partial \sigma_{\theta}}{\partial \theta}+\frac{\partial\left(r \tau_{r \theta}\right)}{\partial r}+\tau_{r \theta}
\end{array}\right\}+\delta \boldsymbol{\epsilon}^{T} \boldsymbol{\sigma}\right) d \Omega=\left.R \int_{\theta_{s}}^{\theta_{e}}\left(\delta \mathbf{u}^{T}\left\{\begin{array}{c}
\sigma_{r} \\
\tau_{r \theta}
\end{array}\right\}\right)\right|_{r=R} d \theta+\left.\int_{0}^{R}\left(\delta \mathbf{u}^{T}\left\{\begin{array}{c}
\tau_{r \theta} \\
\sigma_{\theta}
\end{array}\right\}\right)\right|_{\theta=\theta_{s}} ^{\theta=\theta_{e}} d r
$$

\section{GOVERNING EQUATIONS FOR 2/D PROBLEMS AND THEIR WEAK FORM}

Fig.2b shows a sectorial bimaterial domain formed by two subdomains $\Omega^{A}$ and $\Omega^{B}$. The field variables defined in $\Omega^{A}$ and $\Omega^{B}$ will be followed by superscripts "A" and "B", respectively. Governing equations for the bimaterial domain include :

(a) homogeneous stress equilibrium conditions (see Eqn.(2))

(b) traction-free conditions: $\left.\quad \sigma_{\theta}^{A}\right|_{\theta=\theta_{s}}=\left.\tau_{r \theta}^{A}\right|_{\theta=\theta_{s}}=\left.\sigma_{\theta}^{B}\right|_{\theta=\theta_{e}}=\left.\tau_{r \theta}^{B}\right|_{\theta=\theta_{e}}=0$

(c) traction reciprocity conditions: $\left.\left(\sigma_{\theta}^{A}-\sigma_{\theta}^{B}\right)\right|_{\theta=\theta_{A B}}=\left.\left(\tau_{r \theta}^{A}-\tau_{r \theta}^{B}\right)\right|_{\theta=\theta_{A B}}=0$

(d) displacement compatibility conditions: $\left.\left(\mathbf{u}^{A}-\mathbf{u}^{B}\right)\right|_{\theta=\theta_{A B}}=\mathbf{0}$

in which $\theta_{A B}$ is the angular coordinate of the interface between $\Omega^{A}$ and $\Omega^{B}$. For the purpose of determining the eigenpair, no condition has be to imposed along the arc at $r=R$. Assuming that the displacement compatibility conditions are satisfied as a priori and thus

$$
\left.\left(\delta \mathbf{u}^{A}-\delta \mathbf{u}^{B}\right)\right|_{\theta=\theta_{A B}}=\mathbf{0},
$$

following is a weak form of the governing equations (2), (5) and (6) :

$$
\begin{aligned}
& -\int_{\Omega^{A}} \frac{1}{r}\left(\delta \mathbf{u}^{A}\right)^{T}\left\{\begin{array}{l}
\frac{\partial\left(r \sigma_{r}^{A}\right)}{\partial r}+\frac{\partial \tau_{r \theta}^{A}}{\partial \theta}-\sigma_{\theta}^{A} \\
\frac{\partial \sigma_{\theta}^{A}}{\partial \theta}+\frac{\partial\left(r \tau_{r \theta}^{A}\right)}{\partial r}+\tau_{r \theta}^{A}
\end{array}\right\} d \Omega-\int_{\Omega^{B}} \frac{1}{r}\left(\delta \mathbf{u}^{B}\right)^{T}\left\{\begin{array}{c}
\frac{\partial\left(r \sigma_{r}^{B}\right)}{\partial r}+\frac{\partial \tau_{r \theta}^{B}}{\partial \theta}-\sigma_{\theta}^{B} \\
\frac{\partial \sigma_{\theta}^{B}}{\partial \theta}+\frac{\partial\left(r \tau_{r \theta}^{B}\right)}{\partial r}+\tau_{r \theta}^{B}
\end{array}\right\} d \Omega \\
& +\int_{0}^{R}\left[\left.\left(\delta \mathbf{u}^{A}\right)^{T}\left\{\begin{array}{c}
-\tau_{r \theta}^{A} \\
-\sigma_{\theta}^{A}
\end{array}\right\}\right|_{\theta=\theta_{s}} d r+\int_{0}^{R}\left[\left.\left(\delta \mathbf{u}^{A}\right)^{T}\left\{\begin{array}{c}
\tau_{r \theta}^{A}-\tau_{r \theta}^{B} \\
\sigma_{\theta}^{A}-\sigma_{\theta}^{B}
\end{array}\right\}\right|_{\theta=\theta_{A B}} d r+\int_{0}^{R}\left[\left.\left(\delta \mathbf{u}^{B}\right)^{T}\left\{\begin{array}{c}
\tau_{r \theta}^{B} \\
\sigma_{\theta}^{B}
\end{array}\right\}\right|_{\theta=\theta_{e}} d r=0\right.\right.\right.
\end{aligned}
$$

By invoking Eqn.(4), the above weak form becomes :

$$
\int_{\Omega^{A}}\left(\delta \boldsymbol{\epsilon}^{A}\right)^{T} \boldsymbol{\sigma}^{A} d \Omega+\int_{\Omega^{B}}\left(\delta \boldsymbol{\epsilon}^{B}\right)^{T} \boldsymbol{\sigma}^{B} d \Omega-\left.R \int_{\theta_{s}}^{\theta_{A B}}\left[\left(\delta \mathbf{u}^{A}\right)^{T}\left\{\begin{array}{c}
\sigma_{r}^{A} \\
\tau_{r \theta}^{A}
\end{array}\right\}\right]\right|_{r=R} d \theta-\left.R \int_{\theta_{A B}}^{\theta_{e}}\left[\left(\delta \mathbf{u}^{B}\right)^{T}\left\{\begin{array}{c}
\sigma_{r}^{B} \\
\tau_{r \theta}^{B}
\end{array}\right\}\right]\right|_{r=R} d \theta=0
$$

or, simply, 


$$
\int_{\theta_{s}}^{\theta_{e}} \int_{0}^{R} \delta \boldsymbol{\epsilon}^{T} \boldsymbol{\sigma} r d r d \theta-\left.R \int_{\theta_{s}}^{\theta_{e}}\left(\delta \mathbf{u}^{T}\left\{\begin{array}{c}
\sigma_{r} \\
\tau_{r \theta}
\end{array}\right\}\right)\right|_{r=R} d \theta=0
$$

which can be considered as the virtual work principle for eigenpair problem. It is different from the conventional virtual work principle for the sectorial domain in the sense that $\sigma_{r}$ and $\tau_{r \theta}$ in the second term are derived from the displacement instead of being prescribed as the traction boundary condition.

\section{ONE-DIMENSIONAL FINITE ELEMENT FORMULATION FOR 2/D PROBLEMS}

It is trivial to show that the above weak form is also applicable to multi-material domain and thus finite element discretization. To fulfill the compatibility condition, the displacement at the interfaces of adjacent materials or elements should be maintained at $\mathrm{C}^{0}$. Any higher order continuity would lead to continuous strain and the traction reciprocity can never be satisfied. Let superscript "e" be the designation for a generic element inside which the material is homogeneous. For the element bounded by $\theta_{s}^{e} \leq \theta \leq \theta_{e}^{e}$ and $r=R$, the angular coordinate $\theta$ and its natural coordinate $\xi$ are related as :

$$
\theta=N_{1} \theta_{s}^{e}+N_{2} \theta_{e}^{e} \quad \text { and thus } \quad j^{e}=\frac{d \theta}{d \xi}=\frac{1}{2}\left(\theta_{e}^{e}-\theta_{s}^{e}\right)
$$

in which $N_{1}=(1-\xi) / 2, N_{2}=(1+\xi) / 2$. The $\mathrm{C}^{0}$ hierarchy asymptotic displacements of the element can be expressed as:

$$
\mathbf{u}^{e}=\left\{\begin{array}{c}
u^{e} \\
v^{e}
\end{array}\right\}=r^{\lambda+1} \mathbf{N}^{e}\left\{\begin{array}{lllllllll}
U_{s}^{e} & V_{s}^{e} & \lambda_{U_{1}}^{e} & \lambda_{V_{1}}^{e} & \ldots & \lambda_{U_{n}}^{e} & \lambda_{V_{n}}^{e} & U_{e}^{e} & V_{e}^{e}
\end{array}\right\}^{T}=r^{\lambda+1} \mathbf{N}^{e} \mathbf{q}^{e}
$$

where

$\mathbf{N}^{e}=\left[N_{1} \mathbf{I}_{2}, N_{\lambda 1} \mathbf{I}_{2}, \ldots, N_{\lambda n} \mathbf{I}_{2}, N_{2} \mathbf{I}_{2}\right], N_{\lambda i}=\xi^{i-1}\left(1-\xi^{2}\right), \lambda^{e}$ 's are the coefficients of $N_{\lambda i}$ 's

$\mathbf{q}^{e}$ is the vector containing all the generalized displacement components of the element

$\mathbf{I}_{2}$ is the second order identity matrix

$\left\{U_{s}^{e}, V_{s}^{e}\right\}^{T}$ is the generalized displacement vector at $\theta=\theta_{s}^{e}$

$\left\{U_{e}^{e}, V_{e}^{e}\right\}^{T}$ is the generalized displacement vectors at $\theta=\theta_{e}^{e}$ 
By invoking the strain-displacement relations in Eqn.(1), the element strain and stress can be expressed as :

$$
\boldsymbol{\epsilon}^{e}=r^{\lambda}\left(\lambda \mathbf{B}_{1}^{e}+\mathbf{B}_{0}^{e}\right) \mathbf{q}^{e}, \boldsymbol{\sigma}^{e}=\mathbf{C}^{e} \boldsymbol{\epsilon}^{e}=r^{\lambda} \mathbf{C}^{e}\left(\lambda \mathbf{B}_{1}^{e}+\mathbf{B}_{0}^{e}\right) \mathbf{q}^{e}
$$

in which

$$
\begin{aligned}
& \mathbf{B}_{1}^{e}=\left[\begin{array}{cccccccccc}
N_{1} & 0 & N_{\lambda_{1}} & 0 & \cdots & \cdots & N_{\lambda_{n}} & 0 & N_{2} & 0 \\
0 & 0 & 0 & 0 & \cdots & \cdots & 0 & 0 & 0 & 0 \\
0 & N_{1} & 0 & N_{\lambda_{1}} & \cdots & \cdots & 0 & N_{\lambda_{n}} & 0 & N_{2}
\end{array}\right] \\
& \mathbf{B}_{0}^{e}=\left[\begin{array}{cccccccccc}
N_{1} & 0 & N_{\lambda_{1}} & 0 & \cdots & \cdots & N_{\lambda_{n}} & 0 & N_{2} & 0 \\
N_{1} & N_{1, \theta} & N_{\lambda_{1}} & N_{\lambda_{1}, \theta} & \cdots & \cdots & N_{\lambda_{n}} & N_{\lambda_{n}, \theta} & N_{2} & N_{2, \theta} \\
N_{1, \theta} & 0 & N_{\lambda_{1}, \theta} & 0 & \cdots & \cdots & N_{\lambda_{n}, \theta} & 0 & N_{2, \theta} & 0
\end{array}\right] \\
& \mathbf{C}^{e} \text { is the material stiffness matrix relating } \boldsymbol{\sigma}^{e} \text { and } \boldsymbol{\epsilon}^{e}
\end{aligned}
$$

Thus,

$$
\left\{\begin{array}{c}
\sigma_{r}^{e} \\
\tau_{r \theta}^{e}
\end{array}\right\}=\left[\begin{array}{lll}
1 & 0 & 0 \\
0 & 0 & 1
\end{array}\right] \boldsymbol{\sigma}^{e}=r^{\lambda}\left(\left[\begin{array}{ccc}
1 & 0 & 0 \\
0 & 0 & 1
\end{array}\right] \mathbf{C}^{e}\right)\left(\lambda \mathbf{B}_{1}^{e}+\mathbf{B}_{0}^{e}\right) \mathbf{q}^{e}=r^{\lambda} \mathbf{D}^{e}\left(\lambda \mathbf{B}_{1}^{e}+\mathbf{B}_{0}^{e}\right) \mathbf{q}^{e}
$$

By substituting the above element quantities into Eqn.(10), we have :

$$
\sum_{e}\left[\int_{\theta_{s}^{e}}^{\theta_{e}^{e}} \int_{0}^{R}\left(\delta \boldsymbol{\epsilon}^{e}\right)^{T} \boldsymbol{\sigma}^{e} r d r d \theta\right]-R \sum_{e} \int_{\theta_{s}^{e}}^{\theta_{e}^{e}}\left[\left(\delta \mathbf{u}^{e}\right)^{T}\left\{\begin{array}{c}
\sigma_{r}^{e} \\
\tau_{r \theta}^{e}
\end{array}\right\}\right]_{r=R} d \theta=0
$$

or

$$
\sum_{e}\left[\left(\delta \mathbf{q}^{e}\right)^{T}\left(\lambda^{2} \mathbf{P}^{e}+\lambda \mathbf{Q}^{e}+\mathbf{R}^{e}\right) \mathbf{q}^{e}\right]=0
$$

where

$$
\begin{aligned}
& \mathbf{P}^{e}=\int_{\theta_{s}^{e}}^{\theta_{e}^{e}}\left[\left(\mathbf{B}_{1}^{e}\right)^{T} \mathbf{C}^{e} \mathbf{B}_{1}^{e}-2\left(\mathbf{N}^{e}\right)^{T} \mathbf{D}^{e} \mathbf{B}_{1}^{e}\right] d \theta \quad, \quad \mathbf{R}^{e}=\int_{\theta_{s}^{e}}^{\theta_{e}^{e}}\left[\left(\mathbf{B}_{0}^{e}\right)^{T} \mathbf{C}^{e} \mathbf{B}_{0}^{e}-2\left(\mathbf{N}^{e}\right)^{T} \mathbf{D}^{e} \mathbf{B}_{0}^{e}\right] d \theta \\
& \mathbf{Q}^{e}=\int_{\theta_{s}^{e}}^{\theta_{e}^{e}}\left[\left(\mathbf{B}_{1}^{e}\right)^{T} \mathbf{C}^{e} \mathbf{B}_{0}^{e}+\left(\mathbf{B}_{0}^{e}\right)^{T} \mathbf{C}^{e} \mathbf{B}_{1}^{e}-2\left(\mathbf{N}^{e}\right)^{T} \mathbf{D}^{e}\left(\mathbf{B}_{0}^{e}+\mathbf{B}_{1}^{e}\right)\right] d \theta
\end{aligned}
$$

Owing to the arbitrary nature of $\delta \mathbf{q}^{e}$ 's, a second order characteristic equation of the following form can be obtained :

$$
\left(\lambda^{2} \mathbf{P}+\lambda \mathbf{Q}+\mathbf{R}\right) \mathbf{q}=0
$$

in which $\mathbf{q}$ is the collection of all $\mathbf{q}^{e}$ 's, matrices $\mathbf{P}, \mathbf{Q}$ and $\mathbf{R}$ are the assembled counterparts of $\mathbf{P}^{e}$, $\mathrm{s}$, 
$\mathbf{Q}^{e}$ 's and $\mathbf{R}^{e}$ 's, respectively.

Eqn.(16) can be transformed into the standard first order characteristic equation by introducing a dummy vector $\tilde{\mathbf{q}}=\lambda \mathbf{q}$ with which Eqn.(16) can be expressed as :

$$
\left[\begin{array}{cc}
-\mathbf{P}^{-1} \mathbf{Q} & -\mathbf{P}^{-1} \mathbf{R} \\
\mathbf{I} & \mathbf{0}
\end{array}\right]\left\{\begin{array}{c}
\tilde{\mathbf{q}} \\
\mathbf{q}
\end{array}\right\}=\lambda\left\{\begin{array}{c}
\tilde{\mathbf{q}} \\
\mathbf{q}
\end{array}\right\}
$$

which can be solved by standard numerical subroutines for eigenvalue problems.

\section{NUMERICAL EXAMPLES FOR 2/D SECTORIAL PROBLEMS}

In this section, several numerical examples are presented. The materials involved are all isotropic. With reference to Eqn.(16), it is trivial that if $\lambda$ is an eigenvalue, so is its complex congugate. While the $\lambda$ 's with their real parts greater than zero do not induce stress singularities, the ones with their real parts less than -1 lead to infinite strain energy density and thus are non-physical. Only the $\lambda$ 's whose real parts are within -1 and zero are of concern and reported here.

5.1 Crack in Isotropic Material - A crack in an isotropic material with $v=0.3$ and under plane stress condition is considered. The exact solutions of $\lambda$ for this problem are $-1 / 2$ for both mode I and mode II fractures. The whole circumferential domain from $-\pi$ to $+\pi$ is modelled by two equalsized elements, see Fig.1c, and the numbers of bubble modes employed in the two elements are identical. Fig.3 shows that both the present and Pageau's [2] predictions exhibit strong and monotonic convergence toward the exact solution.

5.2 Interfacial Crack - The interfacial crack problem as shown in Fig.1c is considered. The analytical solution of this problem were obtained by Williams [8], Rice \& Sih [9], England [10], etc. to be :

$$
\lambda=-\frac{1}{2} \pm \frac{i}{2 \pi} \ln \left(\frac{k^{A} / G^{A}+1 / G^{B}}{k^{B} / G^{B}+1 / G^{A}}\right)
$$

in which superscipts $A$ and $B$ are the material designations, $G$ is shear modulus, $k=(3-v) /(1+v)$ for plane stress condition and $k=3-4 v$ for plain strain condition. Following Gu \& Belytschko's settings, $v^{A}=v^{B}=0.3$. The imaginary part of $\lambda$ leads to stress oscillations at the vicinity of the crack tip. Table 1 lists the plane stress results by varying the numbers of bubble modes $n_{A}$ and $n_{B}$ in the two elements. With around twenty-two nodal d.o.f. in two elements, the present results are 
comparable in accuracy with the ones obtained by Gu \& Belytschko's displacement-based method using 162 nodal d.o.f.s in 80 linear elements [7].

Table 1. The computed eigenvalues for 2/D interfacial crack under the plane stress condition. Gu $\&$ Belytschko's results were obtained by their displacement-based method using 162 d.o.f.s

\begin{tabular}{|c|c|c|c|}
\hline$E^{A} / E^{B}$ & present $\left(n_{A}, n_{B}\right.$, no. of d.o.f.s $)$ & exact, see Eqn.(18) & Gu \& Belytschko [7] \\
\hline 1 & $\begin{array}{ll}-0.497192 & (3,3,18) \\
-0.499811 & (4,4,22) \\
-0.499992 & (5,5,26) \\
\end{array}$ & -0.500000 & -0.498500 \\
\hline 2 & $\begin{array}{rr}-0.494312 \pm 0.037840 i & (3,3,18) \\
-0.499628 \pm 0.037388 i & (4,4,22) \\
-0.499984 \pm 0.037310 i & (5,5,26)\end{array}$ & $-0.500000 \pm 0.037306 i$ & $-0.498478+0.056319 i$ \\
\hline 3 & $\begin{array}{rr}-0.494350 \pm 0.057180 i & (3,3,18) \\
-0.499638 \pm 0.056406 i & (4,4,22) \\
-0.499984 \pm 0.056289 i & (5,5,26) \\
\end{array}$ & $-0.500000 \pm 0.056283 i$ & $-0.498478+0.056319 i$ \\
\hline 5 & $\begin{array}{ll}-0.494405 \pm 0.076907 i & (3,3,18) \\
-0.499654 \pm 0.075829 i & (4,4,22) \\
-0.499985 \pm 0.075673 i & (5,5,26)\end{array}$ & $-0.500000 \pm 0.075666 i$ & $-0.498473+0.075712 i$ \\
\hline 10 & $\begin{array}{rr}-0.494468 \pm 0.095324 i & (3,3,18) \\
-0.499672 \pm 0.093974 i & (4,4,22) \\
-0.499986 \pm 0.093783 i & (5,5,26)\end{array}$ & $-0.500000 \pm 0.093774 i$ & $-0.498468+0.093829 i$ \\
\hline 20 & $\begin{array}{ll}-0.494511 \pm 0.106112 i & (3,3,18) \\
-0.499684 \pm 0.104607 i & (4,4,22) \\
-0.499986 \pm 0.104395 i & (5,5,26) \\
\end{array}$ & $-0.500000 \pm 0.104386 i$ & $-0.498465+0.104444 i$ \\
\hline 50 & $\begin{array}{ll}-0.494540 \pm 0.113212 i & (3,3,18) \\
-0.499693 \pm 0.111607 i & (4,4,22) \\
-0.499986 \pm 0.111382 i & (5,5,26)\end{array}$ & $-0.500000 \pm 0.111372 i$ & $-0.498462+0.111433 i$ \\
\hline 100 & 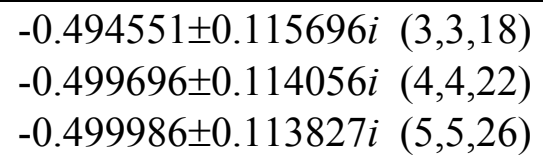 & $-0.500000 \pm 0.113817 i$ & $-0.498461+0.113879 i$ \\
\hline
\end{tabular}

5.3 Bimaterial Wedge - The bimaterial wedge problem as shown in Fig.1b is considered. Material A is bounded by $\theta=-\pi+\alpha$ and $\theta=0$ whereas material $B$ is bounded by $\theta=0$ and $\theta=\pi-\alpha$. Both materials are isotropic and under the plane stress condition. With $E^{A} / E^{B}=100$ and $v^{A}=v^{B}=0.3$, the eigenvalues for various $\alpha$ 's are computed by using 22 nodal d.o.f. in two equal-sized elements $\left(n_{A}=n_{B}=4\right)$ and shown in Fig.4. $\operatorname{Re}(\lambda)$ varies from -0.4997 at $\alpha=0^{\circ}$ to -0.3054 at $\alpha=49^{\circ}$ whereas $\operatorname{Im}(\lambda)$ varies from 0.1140 at $\alpha=0^{\circ}$ to 0 at $\alpha=49^{\circ}$. Within $\alpha=49^{\circ}$ and $\alpha=117^{\circ}$, the eigenvalues are real. For $\alpha \geq 117^{\circ}$, there is no $\lambda$ whose $\operatorname{Re}(\lambda)$ falls between zero and -1 . In other words, the stress would not be singular. The results computed by Gu \& Belytschko's displacementbased method using 162 nodal d.o.f.s in 80 linear elements [7] are also included in the Fig.4. Close agreement can be observed. 
5.4 Bimaterial Free-edge - The bimaterial free-edge problem as shown in Fig.1a is considered. Material $A$ is bounded by $\theta=-\pi / 2$ and $\theta=0$ whereas material $B$ is bounded by $\theta=0$ and $\theta=-\pi / 2$. Both materials are isotropic and $v^{A}=v^{B}=0.3$. When the materials are different, stress singularity will appear. The eigenvalues for different $E^{A}$ to $E^{B}$ ratios are computed by using 22 nodal d.o.f. $\left(n_{A}=n_{B}=4\right)$ in two equal-sized elements and listed in Table 2 for both plane stress and plane strain conditions. The results obtained by $\mathrm{Gu} \&$ Belytschko's stress function-based method are also included for comparison. For their results, the employed number of nodal d.o.f.s is also 22. As the characteristic equation resulted from their method is fourth order, the size of the equivalent first order equation is thus 88 whereas that of the present method is only 44 . Considerable differences can be noted in Table 2. In the case of material A being rigid, i.e. $E^{A} / E^{B}=\infty$, the eigenvalue obtained by solution Williams [11] and Hein \& Erdogan [12] is -0.289 which agrees well with the present and Gu \& Belytschko's predictions at $E^{A} / E^{B}=5000$.

Table 2. The computed eigenvalues for 2/D free-edge problem. Gu \& Belystcho's results were obtained by their stress function-based method

\begin{tabular}{|c|c|c|c|c|}
\hline \multirow{2}{*}{$E^{A} / E^{B}$} & \multicolumn{2}{|c|}{ plane stress condition } & \multicolumn{2}{c|}{ plane strain condition } \\
\cline { 2 - 5 } & present & Gu \& Belytschko [7] & present & Gu \& Belytschko [7] \\
\hline 2 & -0.02670 & -0.09185 & -0.03700 & -0.10653 \\
\hline 5 & -0.10550 & -0.17059 & -0.13618 & -0.20111 \\
\hline 10 & -0.15918 & -0.2035 & -0.19846 & -0.24148 \\
\hline 20 & -0.19570 & -0.22173 & -0.23915 & -0.26406 \\
\hline 50 & -0.22193 & -0.23334 & -0.26770 & -0.27851 \\
\hline 100 & -0.23154 & -0.23734 & -0.27803 & -0.28549 \\
\hline 200 & -0.23653 & -0.23936 & -0.28337 & -0.28602 \\
\hline 5000 & -0.24144 & -0.24132 & -0.28861 & -0.28853 \\
\hline
\end{tabular}

5.5 Bonded Bimaterial Junction - Fig.1d shows the problem of a composite body consisting of a $90^{\circ}$ and a $270^{\circ}$ isotropic material wedges. The two wedges are perfectly bonded along the two interfaces. This kind of configurations can be found inside electronic packages. The plane stress condition is assumed and $v^{A}=v^{B}=0.3$. In the finite element formulation, the traction reciprocity condition at $\theta=\theta_{s}=\theta_{e}$ can be enforced by using the same nodal d.o.f.s at the same angular coordinate. The eigenvalues for various $E^{A}$ to $E^{B}$ ratios are computed by using 28 nodal d.o.f. $\left(n_{A}=n_{B}=6\right)$ in two elements and ploted in Fig.5. The analytical solutions obtained by Chen \& Nisitani are also included for comparison [13]. Close agreement can be noted.

5.6 Debonded Bimaterial Junction - Fig.1e shows the configuration when the interface at $\theta=-\pi$ in Fig.1d is debonded. The eigenvalues for various $E^{A}$ to $E^{B}$ ratios are computed by using 30 nodal 
d.o.f. ( $\left.n_{A}=n_{B}=6\right)$ in two elements and ploted in Fig.6. Again, close agreement is observed for the present results and those obtained by Chen \& Nisitani [13].

\section{DIVERGENCE THEOREM FOR 3/D SECTORIAL DOMAIN}

A 3/D sectorial domain as shown in Fig. 8 is best described by the cylinderical coordinates $(r, \theta, z)$ with respect to which the strain-displacement relation are [14] :

$$
\boldsymbol{\epsilon}=\left\{\varepsilon_{r}, \varepsilon_{\theta}, \varepsilon_{z}, \gamma_{r \theta}, \gamma_{\theta z}, \gamma_{z r}\right\}^{T}=\left\{\frac{\partial u}{\partial r}, \frac{u}{r}+\frac{1}{r} \frac{\partial v}{\partial \theta}, \frac{\partial w}{\partial z}, \frac{1}{r} \frac{\partial u}{\partial \theta}+r \frac{\partial}{\partial r}\left(\frac{v}{r}\right), \frac{\partial v}{\partial z}+\frac{1}{r} \frac{\partial w}{\partial \theta}, \frac{\partial w}{\partial r}+\frac{\partial u}{\partial z}\right\}^{T}
$$

in which $u, v$ and $w$ are the radial, circumferential and longitudinal displacements. On the other hand, the homogeneous equilibrium conditions are :

$$
\frac{\partial\left(r \sigma_{r}\right)}{\partial r}+\frac{\partial \tau_{r \theta}}{\partial \theta}+\frac{\partial\left(r \tau_{z r}\right)}{\partial z}-\sigma_{\theta}=0, \frac{\partial \sigma_{\theta}}{\partial \theta}+\frac{\partial\left(r \tau_{r \theta}\right)}{\partial r}+\frac{\partial\left(r \tau_{\theta z}\right)}{\partial z}+\tau_{r \theta}=0, \frac{\partial\left(r \tau_{z r}\right)}{\partial r}+\frac{\partial \tau_{z \theta}}{\partial \theta}+\frac{\partial\left(r \sigma_{z}\right)}{\partial z}=0
$$

It is a straight forward matter to prove the following identity :

$$
\begin{gathered}
\frac{1}{r} \delta \mathbf{u}^{T}\left\{\begin{array}{c}
\frac{\partial\left(r \sigma_{r}\right)}{\partial r}+\frac{\partial \tau_{r \theta}}{\partial \theta}+\frac{\partial\left(r \tau_{z r}\right)}{\partial z}-\sigma_{\theta} \\
\frac{\partial \sigma_{\theta}}{\partial \theta}+\frac{\partial\left(r \tau_{r \theta}\right)}{\partial r}+\frac{\partial\left(r \tau_{\theta z}\right)}{\partial z}+\tau_{r \theta} \\
\frac{\partial\left(r \tau_{z r}\right)}{\partial r}+\frac{\partial \tau_{z \theta}}{\partial \theta}+\frac{\partial\left(r \sigma_{z}\right)}{\partial z}
\end{array}\right\}+\delta \boldsymbol{\epsilon}^{T} \boldsymbol{\sigma} \\
=\frac{1}{r}\left[\frac{\partial}{\partial r}\left(r \delta \mathbf{u}^{T}\left\{\begin{array}{l}
\sigma_{r} \\
\tau_{r \theta} \\
\tau_{z r}
\end{array}\right\}\right)+\frac{\partial}{\partial \theta}\left(\delta \mathbf{u}^{T}\left\{\begin{array}{l}
\tau_{r \theta} \\
\sigma_{\theta} \\
\tau_{\theta z}
\end{array}\right\}\right)+\frac{\partial}{\partial z}\left(r \delta \mathbf{u}^{T}\left\{\begin{array}{l}
\tau_{z r} \\
\tau_{\theta z} \\
\sigma_{z}
\end{array}\right\}\right)\right]
\end{gathered}
$$

where $\mathbf{u}=\{u, v, w\}^{T}$ and $\boldsymbol{\sigma}=\left\{\sigma_{r}, \sigma_{\theta}, \sigma_{z}, \tau_{r \theta}, \tau_{\theta z}, \tau_{z r}\right\}^{T}$ are the vectors of displacement and stress components. By integrating Eqn.(21) over the three-dimensional sectorial domain $\Omega$ bounded by $\theta=\theta_{s}, \theta=\theta_{e}, z=z_{s}, z=z_{e}$ and $r=R$, we have

$$
\int_{\Omega}\left(\frac{1}{r} \delta \mathbf{u}^{T}\left\{\begin{array}{c}
\frac{\partial\left(r \sigma_{r}\right)}{\partial r}+\frac{\partial \tau_{r \theta}}{\partial \theta}+\frac{\partial\left(r \tau_{z r}\right)}{\partial z}-\sigma_{\theta} \\
\frac{\partial \sigma_{\theta}}{\partial \theta}+\frac{\partial\left(r \tau_{r \theta}\right)}{\partial r}+\frac{\partial\left(r \tau_{\theta z}\right)}{\partial z}+\tau_{r \theta} \\
\frac{\partial\left(r \tau_{z r}\right)}{\partial r}+\frac{\partial \tau_{z \theta}}{\partial \theta}+\frac{\partial\left(r \sigma_{z}\right)}{\partial z}
\end{array}\right\}+\delta \boldsymbol{\epsilon}^{T} \boldsymbol{\sigma}\right) d \Omega
$$




$$
=\left.R \int_{z_{s}}^{z_{e}} \int_{\theta_{s}}^{\theta_{e}}\left(\delta \mathbf{u}^{T}\left\{\begin{array}{c}
\sigma_{r} \\
\tau_{r \theta} \\
\tau_{r z}
\end{array}\right\}\right)\right|_{r=R} d \theta d z+\left.\int_{0}^{R} \int_{z_{s}}^{z_{e}}\left(\delta \mathbf{u}^{T}\left\{\begin{array}{c}
\tau_{r \theta} \\
\sigma_{\theta} \\
\tau_{\theta z}
\end{array}\right\}\right)\right|_{\theta=\theta_{s}} ^{\theta=\theta_{e}} d z d r+\left.\int_{\theta_{s}}^{\theta_{e}} \int_{0}^{R}\left(r \delta \mathbf{u}^{T}\left\{\begin{array}{l}
\tau_{r z} \\
\tau_{\theta z} \\
\sigma_{z}
\end{array}\right\}\right)\right|_{z=z_{s}} ^{z=z_{e}} d r d \theta
$$

\section{GOVERNING EQUATIONS FOR 3/D PROBLEMS AND THEIR WEAK FORM}

For a bimaterial sectorial domain formed by subdomains $\Omega^{A}$ and $\Omega^{B}$ as depicted in Fig.2b, the governing equations for the bimaterial domain include:

(a) homogeneous stress equilibrium conditions (see Eqn.(20))

(b) traction-free conditions:

$$
\left\{\begin{array}{c}
\tau_{r \theta}^{A} \\
\sigma_{\theta}^{A} \\
\tau_{\theta z}^{A}
\end{array}||_{\theta=\theta_{s}}=\left\{\begin{array}{c}
\tau_{z r}^{A} \\
\tau_{\theta z}^{A} \\
\sigma_{z}^{A}
\end{array}||_{z=z_{s}}=\left.\left\{\begin{array}{c}
\tau_{z r}^{A} \\
\tau_{\theta z}^{A} \\
\sigma_{z}^{A}
\end{array}\right\}\right|_{z=z_{e}}=\left.\left\{\begin{array}{c}
\tau_{r \theta}^{B} \\
\sigma_{\theta}^{B} \\
\tau_{\theta z}^{B}
\end{array}\right\}\right|_{\theta=\theta_{s}}=\left.\left\{\begin{array}{c}
\tau_{z r}^{B} \\
\tau_{\theta z}^{B} \\
\sigma_{z}^{B}
\end{array}\right\}\right|_{z=z_{s}}=\left\{\begin{array}{c}
\tau_{z r}^{B} \\
\tau_{z_{z}}^{B} \\
\sigma_{z}^{B}
\end{array}||_{z=z_{e}}=\mathbf{0}\right.\right.\right.
$$

(c) traction reciprocity conditions: $\left(\left\{\begin{array}{c}\tau_{r \theta}^{A} \\ \sigma_{\theta}^{A} \\ \tau_{\theta z}^{A}\end{array}\right\}-\left.\left\{\begin{array}{c}\tau_{r \theta}^{B} \\ \sigma_{\theta}^{B} \\ \tau_{\theta z}^{B}\end{array}\right\}\right|_{\theta=\theta_{A B}}=\mathbf{0}\right.$

(d) displacement compatibility conditions: $\left.\left(\mathbf{u}^{A}-\mathbf{u}^{B}\right)\right|_{\theta=\theta_{A B}}=\mathbf{0}$

Assuming the displacement compatibility conditions are satisfied as a priori and thus

$$
\left.\left(\delta \mathbf{u}^{A}-\delta \mathbf{u}^{B}\right)\right|_{\theta=\theta_{A B}}=\mathbf{0},
$$

the following is a weak form of the governing equations (20), (23) and (24):

$$
\begin{gathered}
-\int_{\Omega^{A}} \frac{1}{r} \delta\left(\mathbf{u}^{A}\right)^{T}\left\{\begin{array}{c}
\frac{\partial\left(r \sigma_{r}^{A}\right)}{\partial r}+\frac{\partial \tau_{r \theta}^{A}}{\partial \theta}+\frac{\partial\left(r \tau_{z r}^{A}\right)}{\partial z}-\sigma_{\theta}^{A} \\
\frac{\partial \sigma_{\theta}^{A}}{\partial \theta}+\frac{\partial\left(r \tau_{r \theta}^{A}\right)}{\partial r}+\frac{\partial\left(r \tau_{\theta z}^{A}\right)}{\partial z}+\tau_{r \theta}^{A} \\
\frac{\partial\left(r \tau_{z r}^{A}\right)}{\partial r}+\frac{\partial \tau_{z \theta}^{A}}{\partial \theta}+\frac{\partial\left(r \sigma_{z}^{A}\right)}{\partial z}
\end{array}\right\} d \Omega-\int_{\Omega^{B}} \frac{1}{r} \delta\left(\mathbf{u}^{B}\right)^{T}\left\{\begin{array}{c}
\frac{\partial\left(r \sigma_{r}^{B}\right)}{\partial r}+\frac{\partial \tau_{r \theta}^{B}}{\partial \theta}+\frac{\partial\left(r \tau_{z r}^{B}\right)}{\partial z}-\sigma_{\theta}^{B} \\
\frac{\partial \sigma_{\theta}^{B}}{\partial \theta}+\frac{\partial\left(r \tau_{r \theta}^{B}\right)}{\partial r}+\frac{\partial\left(r \tau_{\theta z}^{B}\right)}{\partial z}+\tau_{r \theta}^{B} \\
\frac{\partial\left(r \tau_{z r}^{B}\right)}{\partial r}+\frac{\partial \tau_{z \theta}^{B}}{\partial \theta}+\frac{\partial\left(r \sigma_{z}^{B}\right)}{\partial z}
\end{array}\right\} d \Omega \\
-\int_{0}^{R} \int_{z_{s}}^{z_{e}}\left(\left.\delta \mathbf{u}^{T}\left\{\begin{array}{l}
\tau_{r \theta}^{A} \\
\sigma_{\theta}^{A} \\
\tau_{\theta z}^{A}
\end{array}\right)\right|_{\theta=\theta_{s}} d z d r+\int_{0}^{R} \int_{z_{s}}^{z_{e}}\left(\left.\delta \mathbf{u}^{T}\left\{\begin{array}{c}
\tau_{r \theta}^{A}-\tau_{r \theta}^{B} \\
\sigma_{\theta}^{A}-\sigma_{\theta}^{B} \\
\tau_{\theta z}^{A}-\tau_{\theta z}^{B}
\end{array}\right)\right|_{\theta=\theta_{A B}} d z d r+\left.\int_{0}^{R} \int_{z_{s}}^{z_{e}}\left(\delta \mathbf{u}^{T}\left\{\begin{array}{l}
\tau_{r \theta}^{B} \\
\sigma_{\theta}^{B} \\
\tau_{\theta z}^{B}
\end{array}\right)\right)\right|_{\theta=\theta_{e}} d z d r\right.\right.
\end{gathered}
$$




$$
\left.+\left.\int_{\theta_{s}}^{\theta_{A B}} \int_{0}^{R}\left[r\left(\delta \mathbf{u}^{A}\right)^{T}\left\{\begin{array}{c}
\tau_{z r}^{A} \\
\tau_{\theta z}^{A} \\
\sigma_{z}^{A}
\end{array}\right\}\right]\right|_{z=z_{s}} ^{z=z_{e}} d r d \theta+\int_{\theta_{A B}}^{\theta_{e}} \int_{0}^{R}\left[r\left(\delta \mathbf{u}^{B}\right)^{T}\left\{\begin{array}{c}
\tau_{z r}^{B} \\
\tau_{\theta z}^{B} \\
\sigma_{z}^{B}
\end{array}\right\}\right]\right)\left.\right|_{z=z_{s}} ^{z=z_{e}} d r d \theta=0
$$

By invoking Eqn.(21), the above weak form becomes:

$$
\begin{aligned}
\int_{\Omega^{A}} \delta\left(\boldsymbol{\epsilon}^{A}\right)^{T} \boldsymbol{\sigma}^{A} d \Omega+\int_{\Omega^{B}} \delta\left(\boldsymbol{\epsilon}^{B}\right)^{T} \boldsymbol{\sigma}^{B} d \Omega & \\
& -R \int_{z_{s}}^{z_{e}} \int_{\theta_{s}}^{\theta_{A B}}\left[\left.\delta\left(\mathbf{u}^{A}\right)^{T}\left\{\begin{array}{c}
\sigma_{r}^{A} \\
\tau_{r \theta}^{A} \\
\tau_{z r}^{A}
\end{array}\right\}\right|_{r=R} d \theta d z-R \int_{z_{s}}^{z_{e}} \int_{\theta_{A B}}^{\theta_{e}}\left[\left.\delta\left(\mathbf{u}^{B}\right)^{T}\left\{\begin{array}{l}
\sigma_{r}^{B} \\
\tau_{r \theta}^{B} \\
\tau_{z r}^{B}
\end{array}\right\}\right|_{r=R} d \theta d z=0\right.\right.
\end{aligned}
$$

which can simply be expressed as :

$$
\int_{z_{s}}^{z_{e}} \int_{\theta_{s}}^{\theta_{e}} \int_{0}^{R} \delta \boldsymbol{\epsilon}^{T} \boldsymbol{\sigma} r d r d \theta d z-\left.R \int_{z_{s}}^{z_{e}} \int_{\theta_{s}}^{\theta_{e}}\left(\delta \mathbf{u}^{T}\left\{\begin{array}{c}
\sigma_{r} \\
\tau_{r \theta} \\
\tau_{z r}
\end{array}\right\}\right)\right|_{r=R} d \theta d z=0
$$

\section{ONE-DIMENSIONAL FINITE ELEMENT FORMULATION FOR 3/D PROBLEMS}

The finite element formulation in the present section is similar to that in Section 4. Same as Gu \& Belytschko [7] in tackling Pipes \& Pagano's problem [15], the following asymptotic form of displacements are assumed :

$$
u=u(r, \theta)=r^{\lambda+1} U(\theta), v=v(r, \theta)=r^{\lambda+1} V(\theta) \text { and } w=w(r, \theta)=r^{\lambda+1} W(\theta)
$$

For finite element formulation, the element displacements, strains and stresses would be :

$$
\begin{aligned}
& \mathbf{u}^{e}=\left\{\begin{array}{c}
u^{e} \\
v^{e} \\
w^{e}
\end{array}\right\}=r^{\lambda+1} \mathbf{N}^{e}\left\{U_{s}^{e}, V_{s}^{e}, W_{s}^{e}, \lambda_{U_{1}}^{e}, \lambda_{V_{1}}^{e}, \lambda_{W_{1}}^{e}, \ldots, \lambda_{U_{n}}^{e}, \lambda_{V_{n}}^{e}, \lambda_{W_{n}}^{e}, U_{e}^{e}, V_{e}^{e}, W_{e}^{e}\right\}^{T}=r^{\lambda+1} \mathbf{N}^{e} \mathbf{q}^{e} \\
& \boldsymbol{\epsilon}^{e}=r^{\lambda}\left(\lambda \mathbf{B}_{1}^{e}+\mathbf{B}_{0}^{e}\right) \mathbf{q}^{e}, \boldsymbol{\sigma}^{e}=\mathbf{C}^{e} \boldsymbol{\epsilon}^{e}=r^{\lambda} \mathbf{C}^{e}\left(\lambda \mathbf{B}_{1}^{e}+\mathbf{B}_{0}^{e}\right) \mathbf{q}^{e}
\end{aligned}
$$

in which

$$
\mathbf{N}^{e}=\left[N_{1} \mathbf{I}_{3}, N_{\lambda 1} \mathbf{I}_{3}, \ldots, N_{\lambda n} \mathbf{I}_{3}, N_{2} \mathbf{I}_{3}\right]
$$




$$
\begin{aligned}
\mathbf{B}_{1}^{e} & =\left[\begin{array}{ccc:ccc:c:ccc:ccc}
N_{1} & 0 & 0 & N_{\lambda 1} & 0 & 0 & \cdots & N_{\lambda_{n}} & 0 & 0 & N_{2} & 0 & 0 \\
0 & 0 & 0 & 0 & 0 & 0 & \cdots & 0 & 0 & 0 & 0 & 0 & 0 \\
0 & 0 & 0 & 0 & 0 & 0 & \cdots & 0 & 0 & 0 & 0 & 0 & 0 \\
0 & N_{1} & 0 & 0 & N_{\lambda 1} & 0 & \cdots & 0 & N_{\lambda_{n}} & 0 & 0 & N_{2} & 0 \\
0 & 0 & 0 & 0 & 0 & 0 & \cdots & 0 & 0 & 0 & 0 & 0 & 0 \\
0 & 0 & N_{1} & 0 & 0 & N_{\lambda 1} & \cdots & 0 & 0 & N_{\lambda_{n}} & 0 & 0 & N_{2}
\end{array}\right] \\
\mathbf{B}_{0}^{e} & =\left[\begin{array}{ccc:ccc:c:cccccc}
N_{1} & 0 & 0 & N_{\lambda_{1}} & 0 & 0 & N_{\lambda_{n}} & 0 & 0 & N_{2} & 0 & 0 \\
N_{1} & N_{1, \theta} & 0 & N_{\lambda_{1}} & N_{\lambda_{1}, \theta} & 0 & \cdots & N_{\lambda_{n}} & N_{\lambda_{n}, \theta} & 0 & N_{2} & N_{2, \theta} & 0 \\
0 & 0 & 0 & 0 & 0 & 0 & \cdots & 0 & 0 & 0 & 0 & 0 & 0 \\
N_{1, \theta} & 0 & 0 & N_{\lambda_{1}, \theta} & 0 & 0 & \cdots & N_{\lambda_{n}, \theta} & 0 & 0 & N_{2, \theta} & 0 & 0 \\
0 & 0 & N_{1, \theta} & 0 & 0 & N_{\lambda_{1}, \theta} & \cdots & 0 & 0 & N_{\lambda_{n}, \theta} & 0 & 0 & N_{2, \theta} \\
0 & 0 & N_{1} & 0 & 0 & N_{\lambda_{1}} & \cdots & 0 & 0 & N_{\lambda_{n}} & 0 & 0 & N_{2}
\end{array}\right]
\end{aligned}
$$

$\mathbf{C}^{e}$ is the material stiffness matrix relating $\boldsymbol{\sigma}^{e}$ and $\boldsymbol{\epsilon}^{e}$ (see Appendix A)

Thus,

$$
\left\{\begin{array}{l}
\sigma_{r}^{e} \\
\tau_{r \theta}^{e} \\
\tau_{z r}^{e}
\end{array}\right\}=\left[\begin{array}{llllll}
1 & 0 & 0 & 0 & 0 & 0 \\
0 & 0 & 0 & 1 & 0 & 0 \\
0 & 0 & 0 & 0 & 0 & 1
\end{array}\right] \boldsymbol{\sigma}^{e}=r^{\lambda}\left(\left[\begin{array}{cccccc}
1 & 0 & 0 & 0 & 0 & 0 \\
0 & 0 & 0 & 1 & 0 & 0 \\
0 & 0 & 0 & 0 & 0 & 1
\end{array}\right] \mathbf{C}^{e}\right)\left(\lambda \mathbf{B}_{1}^{e}+\mathbf{B}_{0}^{e}\right) \mathbf{q}^{e}=r^{\lambda} \mathbf{D}^{e}\left(\lambda \mathbf{B}_{1}^{e}+\mathbf{B}_{0}^{e}\right) \mathbf{q}^{e}
$$

With the last three equations substituted into the weak form in Eqn.(28), the resulting element and global equations are independent of the integration limits for $z$ and of the same form as Eqn.(15) and Eqn.(16). 


\section{NUMERICAL EXAMPLES FOR 3/D SECTORIAL PROBLEMS}

9.1 Crack in Anisotropic Material - A conventional crack in anisotropic material is used here again as a test case. The exact value of $\lambda$ for this problem is again $-1 / 2$ as given by Sih et al [17]. Two equal-sized elements with the same numbers of bubble modes are employed in this problem. Provided that the principal material directions are fixed with respect to the Cartesian coordinate axes, the computed solutions are not sensitive to the material properties. Fig. 8 shows that both the present and Pageau's [3] results converge rapidly toward the exact solution. The present ones are slightly more superior in convergence.

9.2 Free-Edge Problem - The free-edge singularity for a $[ \pm \alpha]$-laminate is investigated, see Fig.9. The laminate is made of high moduli graphite/epoxy laminae with properties : $E_{11}=20.0 \times 10^{6} \mathrm{psi}$, $E_{22}=E_{33}=2.1 \times 10^{6}$ psi, $G_{12}=G_{13}=G_{23}=0.85 \times 10^{6}$ psi and $v_{12}=v_{13}=v_{23}=0.21$ where 1 denotes the fibre running direction. Numerical results of the stress singularity for different $\alpha$ 's are summarized in Table 3 and shown in Fig.10. The problem is modelled by 33 nodal d.o.f.s in two equal-sized elements $\left(n_{A}=n_{B}=4\right)$. Using the present method, the strongest singularity which is found to be -0.026798 and occurs at $\alpha=51^{\circ}$ whereas the strongest stress singularity computed by Gu \& Belytschko is -0.027334 and occurs at $\alpha=53^{\circ}$. At $\alpha=45^{\circ}$, the present result is -0.025517 and Gu \& Belytschko's result is -0.025403 . The former is more accurate with respect to the analytical solution -0.02557 obtained by Wang \& Choi [16].

Table 3. Eigenvalues for $[ \pm \alpha]$ graphite/expoxy laminate. Gu \& Belystcho's results were obtained by their displacement-based method with 123 nodal d.o.f.s in 40 linear elements

\begin{tabular}{|c|c|c|c|c|c|}
\hline fiber orientation, $\alpha$ & $15^{\mathrm{O}}$ & $30^{\mathrm{O}}$ & $45^{\mathrm{O}}$ & $60^{\mathrm{O}}$ & $75^{\mathrm{O}}$ \\
\hline present & -0.000644 & -0.011594 & -0.025517 & -0.023352 & -0.008982 \\
\hline Gu \& Belytschko [7] & -0.001994 & -0.011592 & -0.025403 & -0.020183 & -0.003830 \\
\hline Wang \& Choi [14] & not available & not available & -0.02557 & not available & not available \\
\hline
\end{tabular}

9.3 Bonded Bimaterial Junction - The case of 3/D fully bonded bimaterial junction is considered, see Fig.1d. Both materials A and B are graphite/epoxy whose properties have been given in the last subsection and all the fibers are perpendicular to the y-axis. The fibers in B are making $45^{\circ}$ with the $\mathrm{z}$-axis whereas the angle between the fibers in $\mathrm{A}$ and the $\mathrm{z}$-axis, denoted as $\alpha$, is varied from $0^{\circ}$ to $180^{\circ}$. Fourty-eight nodal d.o.f.s are employed in four equal-sized elements and the number of bubble modes per element is three. Fig.11 shows the computed eigenvalues which are in good agreement with the analytical solutions obtained by Chen [18]. 


\section{CLOSURE}

The governing equations for two- and three-dimensional bimaterial eigenpair problems are reviewed and their weak forms are presented. With the commonly employed asymptotic assumption of the displacements, a one-dimensional finite element formulation is derived that only discretize the displacement circumferentially. A number of numerical benchmark problems have been studied. Finite element convergence can be yielded by either p- or h-refinments. Experience indicated that reasonably accurate solutions can be yielded by using four bubble modes in two to four elements. By making use of the built-in features of the symbolic computational software MAPLE [19], the finite element procedures can be programmed within short MAPLE worksheets. The worksheet prepared for three -dimensional problems can be found in Appendix B.

Acknowledgment - The work described in this paper was substantially supported by a grant from the Research Grant Council of the Hong Kong SAR, P.R.China (Project No. HKU 7083/98E). We wish to thank Dr. Hui Fan (at Nanyang Technological University, Singapore) for the help discussions. 


\section{REFERENCES}

[1] Y.Yamada, H.Okumura, "Finite element analysis of stress and strain singularity eigenstate in inhomogeneous media or composite materials", pp.325-343, Hybrid \& Mixed Finite Element Methods, ed. S.N.Atluri, R.H.Gallagher \& O.C.Zienkiewicz, Wiley, Chichester, 1983

[2] S.S.Pageau, P.F.Joseph, S.B.Biggers, "Finite element analysis of anisotropic materials with singular inplane stress fields", Int.J.Solids Structures, 32, 571-591 (1995)

[3] S.S.Pageau, S.B.Biggers, "A finite element approach to three-dimensional singular stress states in anisotropic multi-material wedges and junctions", Int.J.Solids Structures, 33, 33-47 (1996)

[4] R.S.Barsoum, C.E.Freese, "An iterative approach for the evaluation of delamination stresses in laminated composites”, Inter.J.Numer.Methods Engrg., 20, 1415-1431 (1984)

[5] R.S.Barsoum, "Application of the finite element iterative method to the eigenvalue problem of a crack between dissimilar media", Inter.J.Numer.Methods Engrg., 26, 541-554 (1988)

[6] Z.Yosibash, and B.A.Szabo, "Numerical analysis of singularities in two-dimensions part 1: computation of eigenpairs", Inter.J.Numer.Methods Engrg., 38, 2055-2082 (1995)

[7] L.Gu, T.Belytschko, "A numerical study of stress singularities in a two-material wedge", Int. J.Solids Structures, 31, 865-889 (1994)

[8] M.L.Williams, "The stress around a fault or crack in dissimilar media", Bulletin of the Seismological Society of America, 49, 199-204 (1959)

[9] J.R.Rice, G.C.Sih, "Plane problems of cracks in dissimilar media”, J.Appl.Mech., 32, 418-423 (1965)

[10] A.H.England, “A crack between dissimilar media”, J.Appl.Mech., 32, 400-402 (1965)

[11] M.L.William, "Stress singularities resulting from various boundary conditions in angular coners of plates in extension”, J.Appl.Mech., 19, 526-528 (1952)

[12] V.L.Hein, F.Erdogan, "Stresses singularities in a two-material wedge", Int.J.Fracture Mech., 7, 317-330 (1971)

[13] D.H.Chen, H.Nisitani, "Singular stress field near the corner of jointed dissimilar materials", J.Appl.Mech., 60, 607-613 (1993)

[14] Y.C.Fung, Foundation of Solid Mechanics, Prentice-Hall, New Jersey, 1965

[15] R.B.Pipes, N.J.Pagano, "Interlaminar stresses in composite laminates under uniform axial extension", J.Composite Materials, 4, 538-548 (1970)

[16] S.S.Wang, I.Choi, "The interface crack between dissimilar anisotropic composite material", J.Appl.Mech., 49, 541-548 (1982)

[17] G.C.Sih, P.C.Paris, G.R.Irwin, “On cracks in rectilinearly anisotropic body”, Int.J.Fracture Mech., 1, 189-202 (1965)

[18] H.P.Chen, "Stress singularities in anisotropic multi-material wedges and junctions", Int.J. Solids Structures, 35, 1057-1073 (1998)

[19] A.I.Beltzer, Engineering Analysis with Maple/Mathematica, Academic Press, London, 1995 


\section{Appendix A - Constitutive Relation in Cylindrical Coordinates}

The constitutive relation for a material with respect to its principal directions can be expressed as :

$$
\left\{\begin{array}{c}
\varepsilon_{1} \\
\varepsilon_{2} \\
\varepsilon_{3} \\
\gamma_{12} \\
\gamma_{23} \\
\gamma_{13}
\end{array}\right\}=\mathbf{S}_{P}\left\{\begin{array}{c}
\sigma_{1} \\
\sigma_{2} \\
\sigma_{3} \\
\tau_{12} \\
\tau_{23} \\
\tau_{13}
\end{array}\right\}=\left[\begin{array}{cccccc}
1 / E_{1} & -v_{21} / E_{2} & -v_{31} / E_{3} & 0 & 0 & 0 \\
-v_{12} / E_{1} & 1 / E_{2} & -v_{32} / E_{3} & 0 & 0 & 0 \\
-v_{13} / E_{1} & -v_{23} / E_{2} & 1 / E_{3} & 0 & 0 & 0 \\
0 & 0 & 0 & 1 / G_{12} & 0 & 0 \\
0 & 0 & 0 & 0 & 1 / G_{23} & 0 \\
0 & 0 & 0 & 0 & 0 & 1 / G_{13}
\end{array}\right]\left\{\begin{array}{c}
\sigma_{1} \\
\sigma_{2} \\
\sigma_{3} \\
\tau_{12} \\
\tau_{23} \\
\tau_{13}
\end{array}\right\}
$$

in which 1, 2 and 3 denote the three orthogonal principal material directions, $\mathbf{S}_{P}$ is the prinicpal material compliance matrix, $E_{i}$ 's are the elastic moduli, $v_{i j}$ 's are the Poisson's ratios and $G_{i j}$ 's are the shear moduli. The symmetry of $\mathbf{S}_{P}$ leads to $E_{j} v_{i j}=E_{i} v_{j i}$. The transformation between the strain components in the material principal and Cartesian coordinates is :

$$
\left\{\begin{array}{c}
\varepsilon_{1} \\
\varepsilon_{2} \\
\varepsilon_{3} \\
\gamma_{12} \\
\gamma_{23} \\
\gamma_{13}
\end{array}\right\}=\mathbf{T}\left\{\begin{array}{c}
\varepsilon_{x} \\
\varepsilon_{y} \\
\varepsilon_{z} \\
\gamma_{x y} \\
\gamma_{y z} \\
\gamma_{x z}
\end{array}\right\}=\left[\begin{array}{cccccc}
l_{1}^{2} & m_{1}^{2} & n_{1}^{2} & l_{1} m_{1} & m_{1} n_{1} & l_{1} n_{1} \\
l_{2}^{2} & m_{2}^{2} & n_{2}^{2} & l_{2} m_{2} & m_{2} n_{2} & l_{2} n_{2} \\
l_{3}^{2} & m_{3}^{2} & n_{3}^{2} & l_{3} m_{3} & m_{3} n_{3} & l_{3} n_{3} \\
2 l_{1} l_{2} & 2 m_{1} m_{2} & 2 n_{1} n_{2} & l_{1} m_{2}+l_{2} m_{1} & m_{1} n_{2}+m_{2} n_{1} & n_{1} l_{2}+n_{2} l_{1} \\
2 l_{2} l_{3} & 2 m_{2} m_{3} & 2 n_{2} n_{3} & l_{3} m_{2}+l_{2} m_{3} & m_{3} n_{2}+m_{2} n_{3} & n_{3} l_{2}+n_{2} l_{3} \\
2 l_{1} l_{3} & 2 m_{1} m_{3} & 2 n_{1} n_{3} & l_{1} m_{3}+l_{3} m_{1} & m_{1} n_{3}+m_{3} n_{1} & n_{1} l_{3}+n_{3} l_{1}
\end{array}\right]\left\{\begin{array}{c}
\varepsilon_{x} \\
\varepsilon_{y} \\
\varepsilon_{z} \\
\gamma_{x y} \\
\gamma_{y z} \\
\gamma_{x z}
\end{array}\right\}
$$

where $\left(l_{\mathrm{i}}, m_{\mathrm{i}}, n_{\mathrm{i}}\right)$ are the direction cosines of $i$-th material principal direction. On the other hand, the relation between the strain components in the cylindrical coordinates $(r, \theta, z)$ and Cartessian coordinates $(x, y, z)$, see Fig. 8 , is :

$$
\left\{\begin{array}{c}
\varepsilon_{x} \\
\varepsilon_{y} \\
\varepsilon_{z} \\
\gamma_{x y} \\
\gamma_{y z} \\
\gamma_{x z}
\end{array}\right\}=\tilde{\mathbf{T}}\left\{\begin{array}{c}
\varepsilon_{r} \\
\varepsilon_{\theta} \\
\varepsilon_{z} \\
\gamma_{r \theta} \\
\gamma_{\theta z} \\
\gamma_{r z}
\end{array}\right\}=\left[\begin{array}{cccccc}
c^{2} & s^{2} & 0 & -c s & 0 & 0 \\
s^{2} & c^{2} & 0 & c s & 0 & 0 \\
0 & 0 & 1 & 0 & 0 & 0 \\
2 c s & -2 c s & 0 & c^{2}-s^{2} & 0 & 0 \\
0 & 0 & 0 & 0 & c & s \\
0 & 0 & 0 & 0 & -s & c
\end{array}\right]\left\{\begin{array}{c}
\varepsilon_{r} \\
\varepsilon_{\theta} \\
\varepsilon_{z} \\
\gamma_{r \theta} \\
\gamma_{\theta z} \\
\gamma_{r z}
\end{array}\right\}
$$

in which $c=\cos \theta$ and $s=\sin \theta$. As the strain energy density is an invariant [14], the constitutive relation in cylindrcal coordinates can be derived as :

$$
\left\{\sigma_{r}, \sigma_{\theta}, \sigma_{z}, \tau_{r \theta}, \tau_{\theta z}, \tau_{r z}\right\}^{T}=\tilde{\mathbf{T}}^{T} \mathbf{T}^{T} \mathbf{S}_{p}^{-1} \mathbf{T} \tilde{\mathbf{T}}\left\{\varepsilon_{r}, \varepsilon_{\theta}, \varepsilon_{z}, \gamma_{r \theta}, \gamma_{\theta z}, \gamma_{r z}\right\}^{T}
$$

where the material stiffness matrix is $\mathbf{C}=\tilde{\mathbf{T}}^{T} \mathbf{T}^{T} \mathbf{S}_{P}^{-1} \mathbf{T} \tilde{\mathbf{T}}$. 


\section{Appendix B - Maple Worksheet}

The Maple worksheet is written for 3/D problems. In using the worksheet, the user has to define the following parameters which can be found after “\#\#” :

- the material stiffness matrix for elements $\mathrm{A}$ and $\mathrm{B}: \mathrm{CA}$ and $\mathrm{CB}$ in terms of the angular coordinate ph which is the same as $\theta$

- the numbers of elements in material $\mathrm{A}$ and material $\mathrm{B}$ : neA and neB

- the numbers of bubble modes for elements in material $\mathrm{A}$ and elements in material $\mathrm{B}: \mathrm{nbA}$ and $\mathrm{nbB}$

- define angular coordinates of the boundaries for material A and material B : phetas, phetaAB and phetae

- if a crack, notch or debonded interface is present, set crack $=1$; otherwise, set crack to a different value.

Numerical integration is conducted by using the eleven-point Gaussian Rule which has empirically been found to be sufficient up to four or five bubbles modes for anisotropic materials.

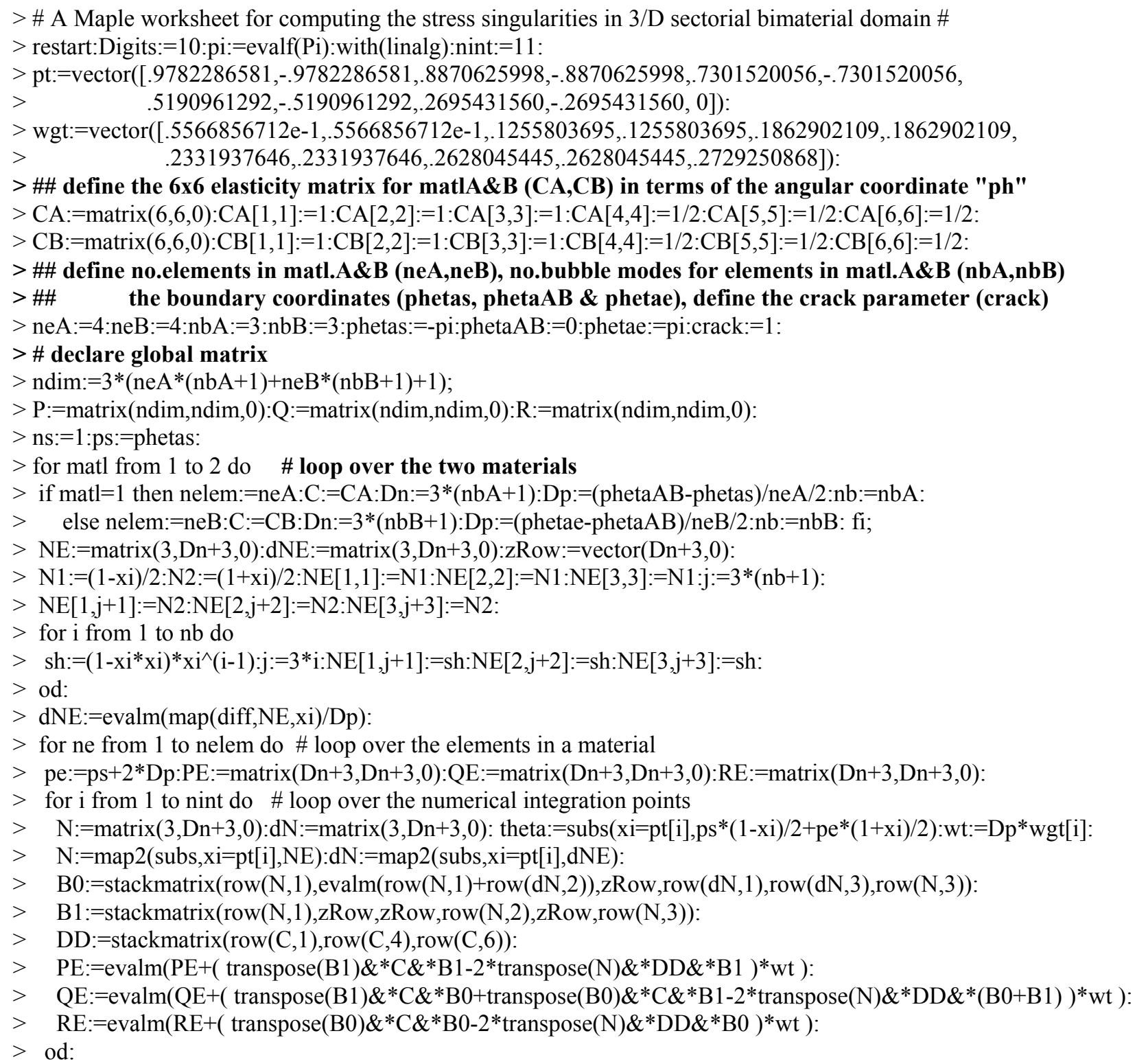




\section{$>$ \# assemble element matrices}

$>\mathrm{PE}[1,1]:=\mathrm{PE}[1,1]+\mathrm{P}[\mathrm{ns}, \mathrm{ns}]: \mathrm{QE}[1,1]:=\mathrm{QE}[1,1]+\mathrm{Q}[\mathrm{ns}, \mathrm{ns}]: \mathrm{RE}[1,1]:=\mathrm{RE}[1,1]+\mathrm{R}[\mathrm{ns}, \mathrm{ns}]$ :

$>\mathrm{PE}[1,2]:=\mathrm{PE}[1,2]+\mathrm{P}[\mathrm{ns}, \mathrm{ns}+1]: \mathrm{QE}[1,2]:=\mathrm{QE}[1,2]+\mathrm{Q}[\mathrm{ns}, \mathrm{ns}+1]: \mathrm{RE}[1,2]:=\mathrm{RE}[1,2]+\mathrm{R}[\mathrm{ns}, \mathrm{ns}+1]$ :

$>\mathrm{PE}[1,3]:=\mathrm{PE}[1,3]+\mathrm{P}[\mathrm{ns}, \mathrm{ns}+2]: \mathrm{QE}[1,3]:=\mathrm{QE}[1,3]+\mathrm{Q}[\mathrm{ns}, \mathrm{ns}+2]: \mathrm{RE}[1,3]:=\mathrm{RE}[1,3]+\mathrm{R}[\mathrm{ns}, \mathrm{ns}+2]:$

$>\mathrm{PE}[2,1]:=\mathrm{PE}[2,1]+\mathrm{P}[\mathrm{ns}+1, \mathrm{~ns}]: \mathrm{QE}[2,1]:=\mathrm{QE}[2,1]+\mathrm{Q}[\mathrm{ns}+1, \mathrm{~ns}]: \mathrm{RE}[2,1]:=\mathrm{RE}[2,1]+\mathrm{R}[\mathrm{ns}+1, \mathrm{~ns}]:$

$>\mathrm{PE}[2,2]:=\mathrm{PE}[2,2]+\mathrm{P}[\mathrm{ns}+1, \mathrm{~ns}+1]: \mathrm{QE}[2,2]:=\mathrm{QE}[2,2]+\mathrm{Q}[\mathrm{ns}+1, \mathrm{~ns}+1]: \mathrm{RE}[2,2]:=\mathrm{RE}[2,2]+\mathrm{R}[\mathrm{ns}+1, \mathrm{~ns}+1]$ :

$>\mathrm{PE}[2,3]:=\mathrm{PE}[2,3]+\mathrm{P}[\mathrm{ns}+1, \mathrm{~ns}+2]: \mathrm{QE}[2,3]:=\mathrm{QE}[2,3]+\mathrm{Q}[\mathrm{ns}+1, \mathrm{~ns}+2]: \mathrm{RE}[2,3]:=\mathrm{RE}[2,3]+\mathrm{R}[\mathrm{ns}+1, \mathrm{~ns}+2]:$

$>\mathrm{PE}[3,1]:=\mathrm{PE}[3,1]+\mathrm{P}[\mathrm{ns}+2, \mathrm{~ns}]: \mathrm{QE}[3,1]:=\mathrm{QE}[3,1]+\mathrm{Q}[\mathrm{ns}+2, \mathrm{~ns}]: \mathrm{RE}[3,1]:=\mathrm{RE}[3,1]+\mathrm{R}[\mathrm{ns}+2, \mathrm{~ns}]$ :

$>\mathrm{PE}[3,2]:=\mathrm{PE}[3,2]+\mathrm{P}[\mathrm{ns}+2, \mathrm{~ns}+1]: \mathrm{QE}[3,2]:=\mathrm{QE}[3,2]+\mathrm{Q}[\mathrm{ns}+2, \mathrm{~ns}+1]: \mathrm{RE}[3,2]:=\mathrm{RE}[3,2]+\mathrm{R}[\mathrm{ns}+2, \mathrm{~ns}+1]$ :

$>\mathrm{PE}[3,3]:=\mathrm{PE}[3,3]+\mathrm{P}[\mathrm{ns}+2, \mathrm{~ns}+2]: \mathrm{QE}[3,3]:=\mathrm{QE}[3,3]+\mathrm{Q}[\mathrm{ns}+2, \mathrm{~ns}+2]: \mathrm{RE}[3,3]:=\mathrm{RE}[3,3]+\mathrm{R}[\mathrm{ns}+2, \mathrm{~ns}+2]:$

$>$ copyinto(PE,P,ns,ns):copyinto(QE,Q,ns,ns):copyinto(RE,R,ns,ns):ps:=pe:ns:=ns+Dn:

$>$ od:

$>$ od:

$>$ \# modify the global matrix when a crack, notch or debonded interface is present

$>$ if crack $<>1$ then

$>$ ndim:=ndim-3:T:=matrix(ndim+3,ndim,0):copyinto(array(1..ndim,1..ndim,identity), $\mathrm{T}, 1,1)$ :

$>$ copyinto(array(1..3,1..3,identity),T,ndim $+1,1) ; \mathrm{P}:=$ evalm(transpose(T)\&*P\&*T):

$>\mathrm{Q}:=$ evalm(transpose $(\mathrm{T}) \& * \mathrm{Q} \& * \mathrm{~T}): \mathrm{R}:=$ evalm(transpose $(\mathrm{T}) \& * \mathrm{R} \& * \mathrm{~T})$ fi:

$>$ \# solve all the eigenvalues and sort out the relevant ones

$>\mathrm{P}$ inv $:=\operatorname{inverse}(\mathrm{P}): \mathrm{PQ}:=\operatorname{multiply}(\mathrm{P}$ inv, $\mathrm{Q}): \mathrm{PR}:=\operatorname{multiply}(\mathrm{P}$ inv, $\mathrm{R})$ :

$>\mathrm{K}_{-}$matrix:=blockmatrix(2,2,[-PQ,-PR,array(1..ndim,1..ndim, identity),matrix(ndim,ndim, 0$\left.)\right]$ ):

$>$ sol:=[eigenvalues $(\mathrm{K}$ matrix $)]$;

$>$ for $\mathrm{i}$ from 1 to $2 *$ ndim do if $\operatorname{Re}(\mathrm{op}(\mathrm{i}, \mathrm{sol}))<0$ and $\operatorname{Re}(\mathrm{op}(\mathrm{i}, \mathrm{sol}))>-1$ then $\operatorname{print}(\mathrm{op}(\mathrm{i}, \mathrm{sol}))$ fi od: 


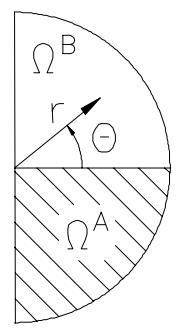

(a)

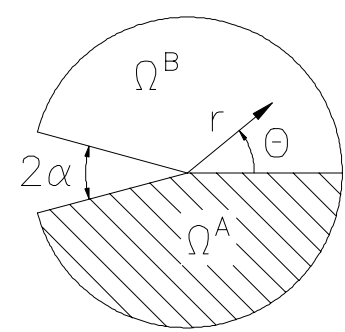

(b)

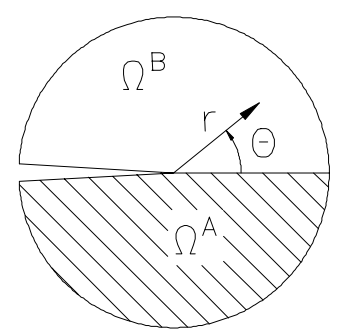

(c)

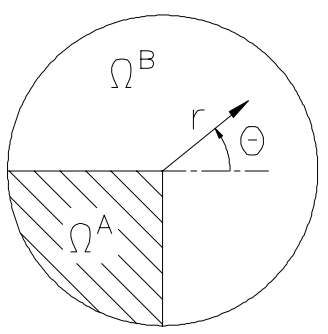

(d)

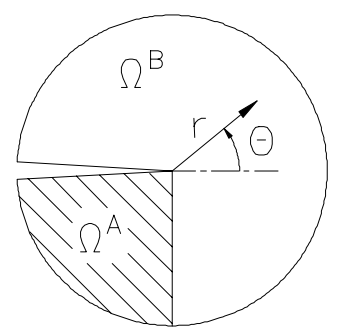

(e)

Fig.1. Examples of stress singularities at bimaterial interfaces : (a) free edge, (b) bimaterial wedge, (c) interfacial crack, (d) bonded bimaterial junction, (e) debonded bimaterial junction

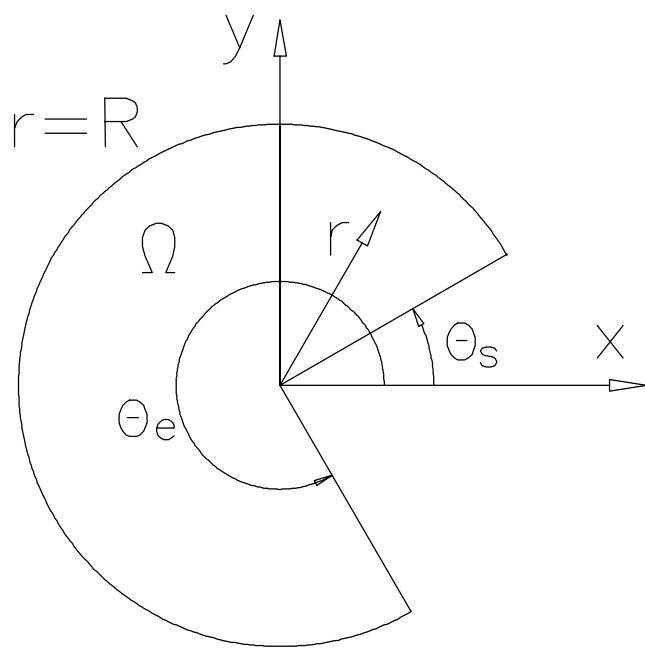

(a)

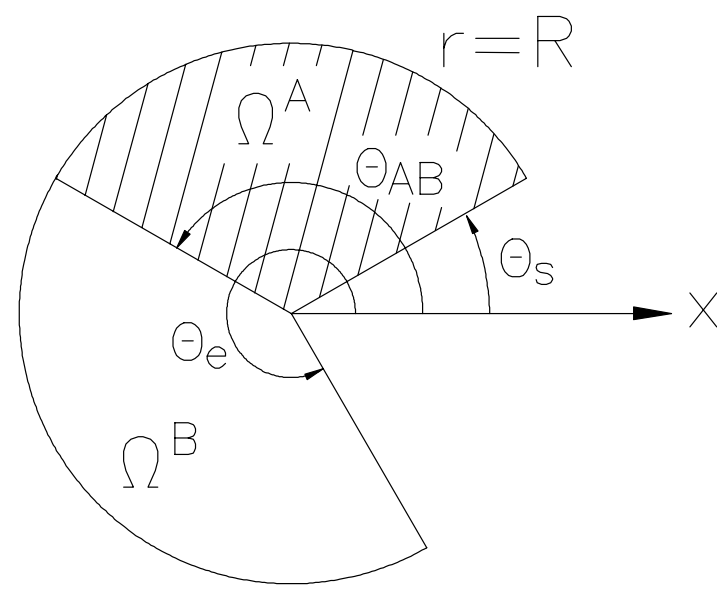

(b)

Fig.2. (a) A single material domain and (b) a bimaterial domain

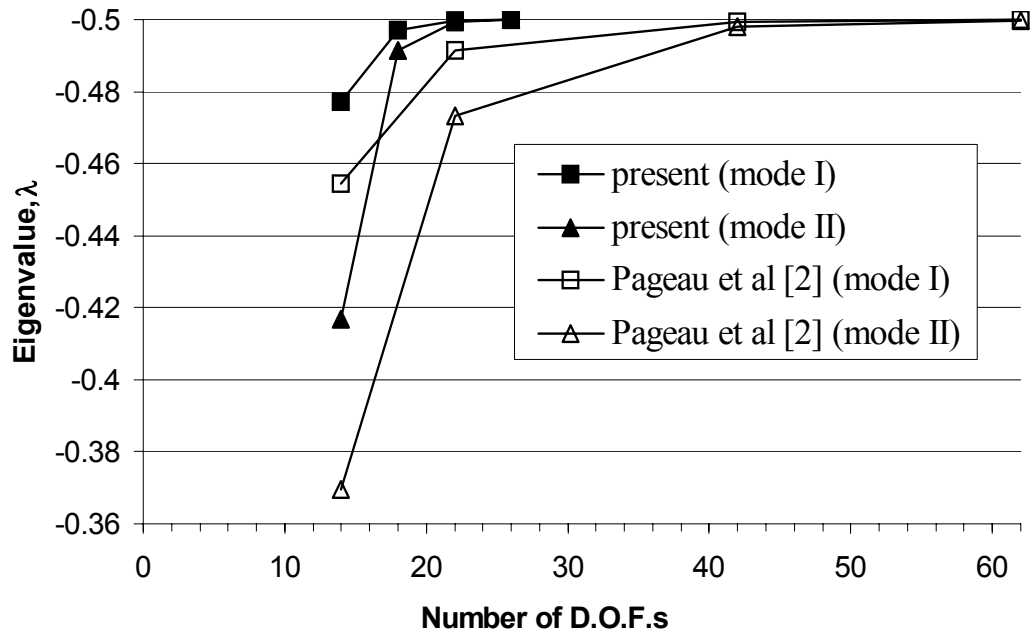

Fig.3. Convergence of the computed eigenvalues for a crack inside an isotropic material 


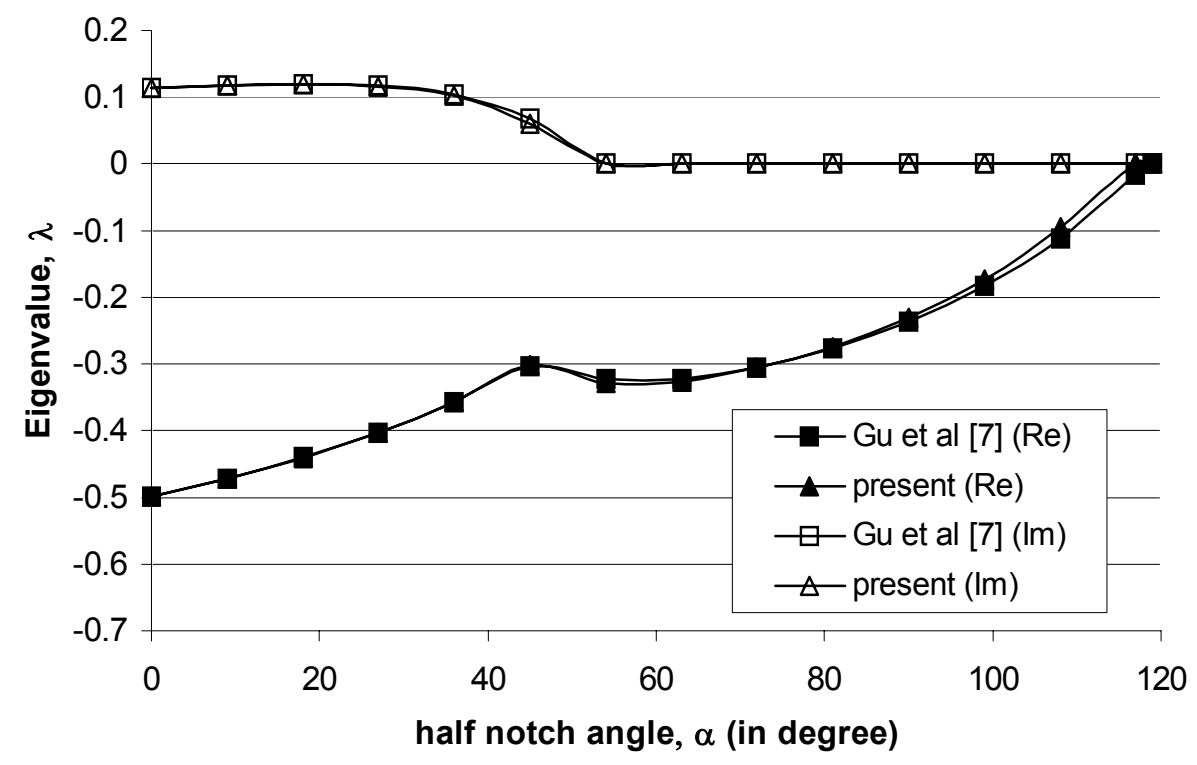

Fig.4. The real and imaginary parts of the computed eigenvalues for bimaterial wedges, see Fig. $1 \mathrm{~b}$

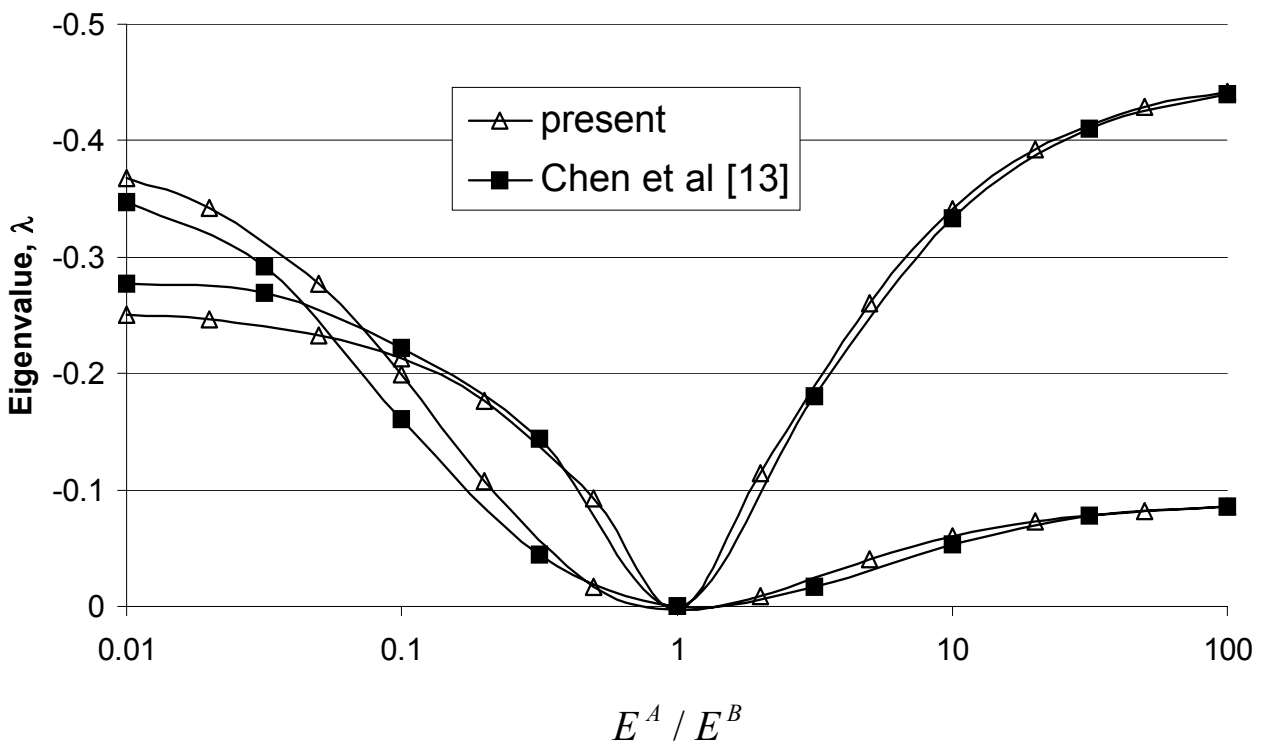

Fig.5. The computed eigenvalues (all real) for bonded bimaterial junctions, see Fig.1d 


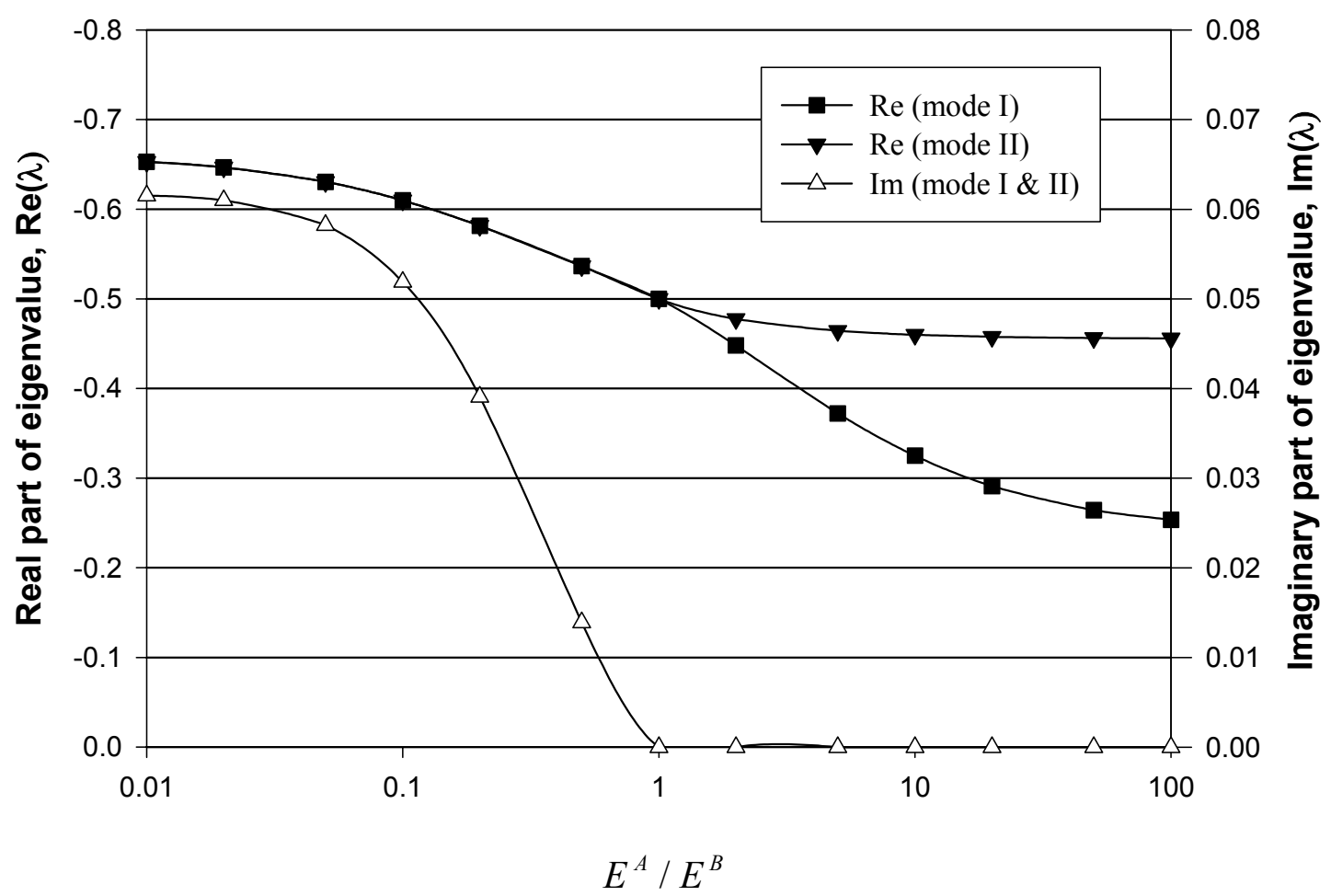

Fig.6. The real and imaginary parts of the computed eigenvalues for debonded bimaterial junctions, see Fig.1e

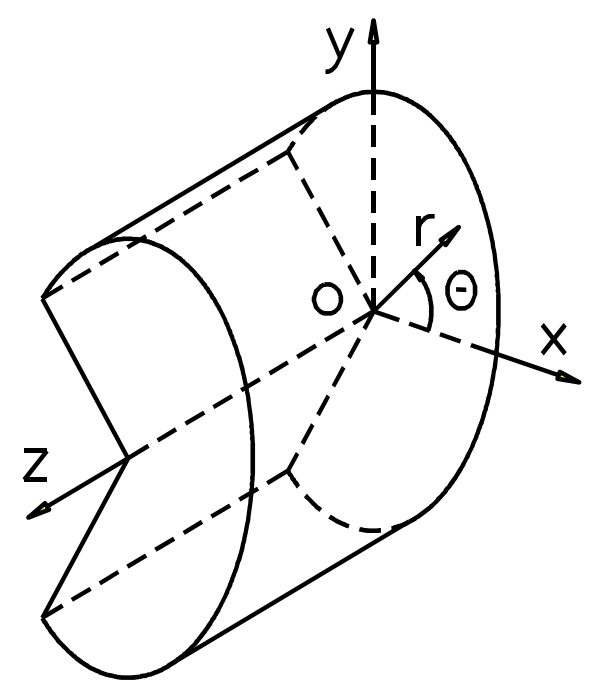

Fig.7. The cylinderical $(r, \theta, z)$ and Cartesian Coordiantes $(x, y, z)$ for a 3/D sectorial domain 


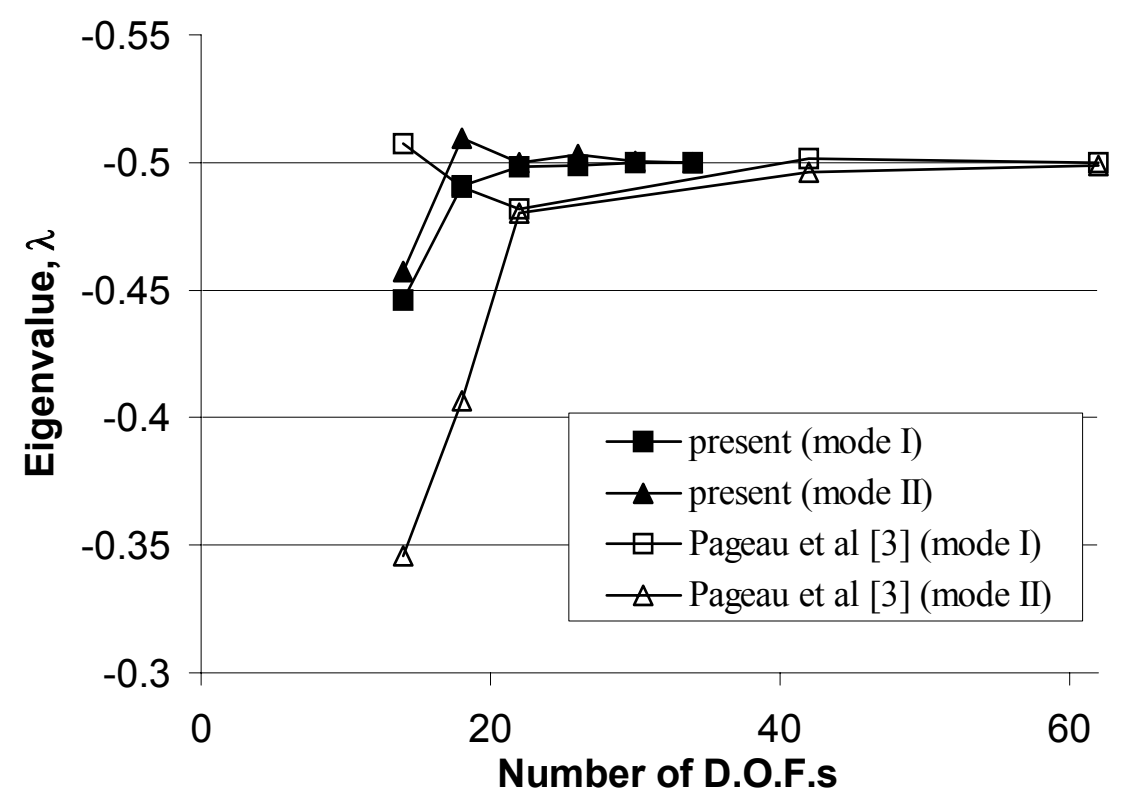

Fig.8. Convergence of the eigenvalues for a crack inside an anisotropic material

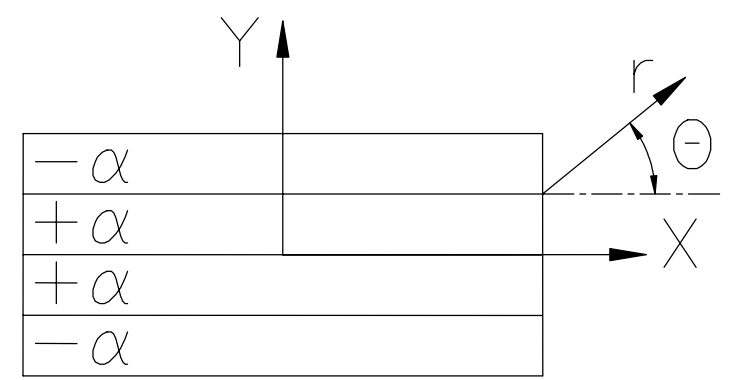

Fig.9. End view of the $[-\alpha / \alpha / \alpha /-\alpha]$ laminate

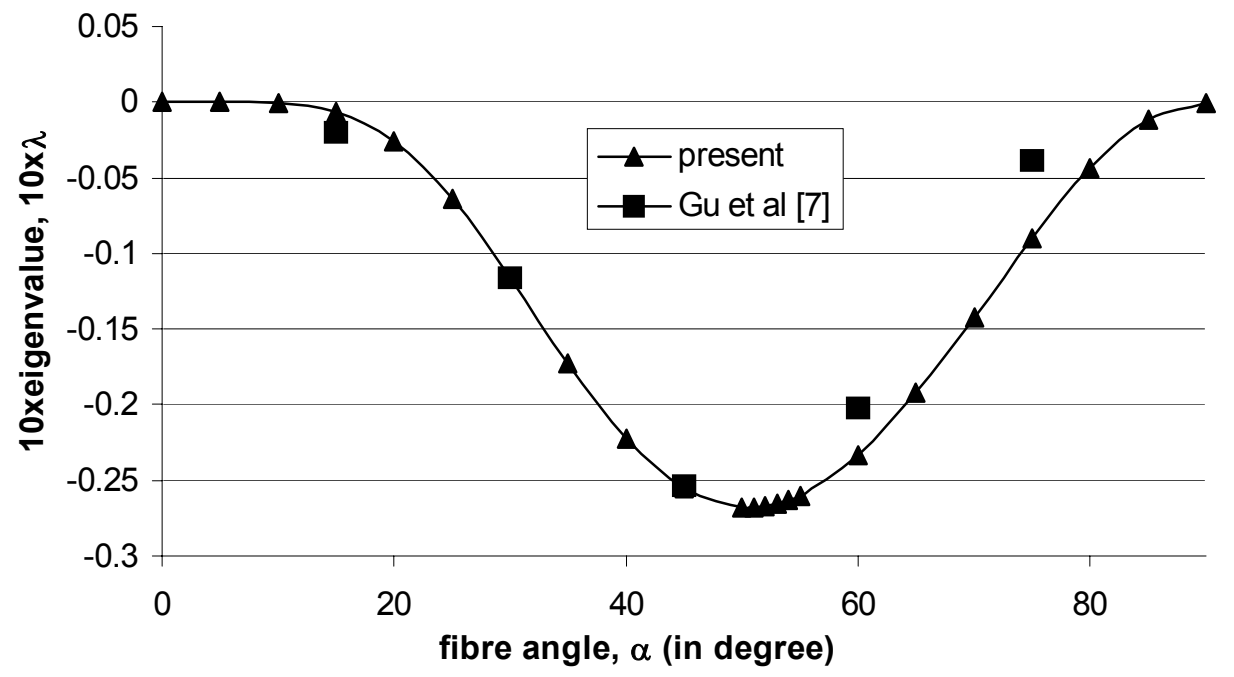

Fig.10. The computed eigenvalues at the free-edge of $[ \pm \alpha]$-laminates, see Fig.9 


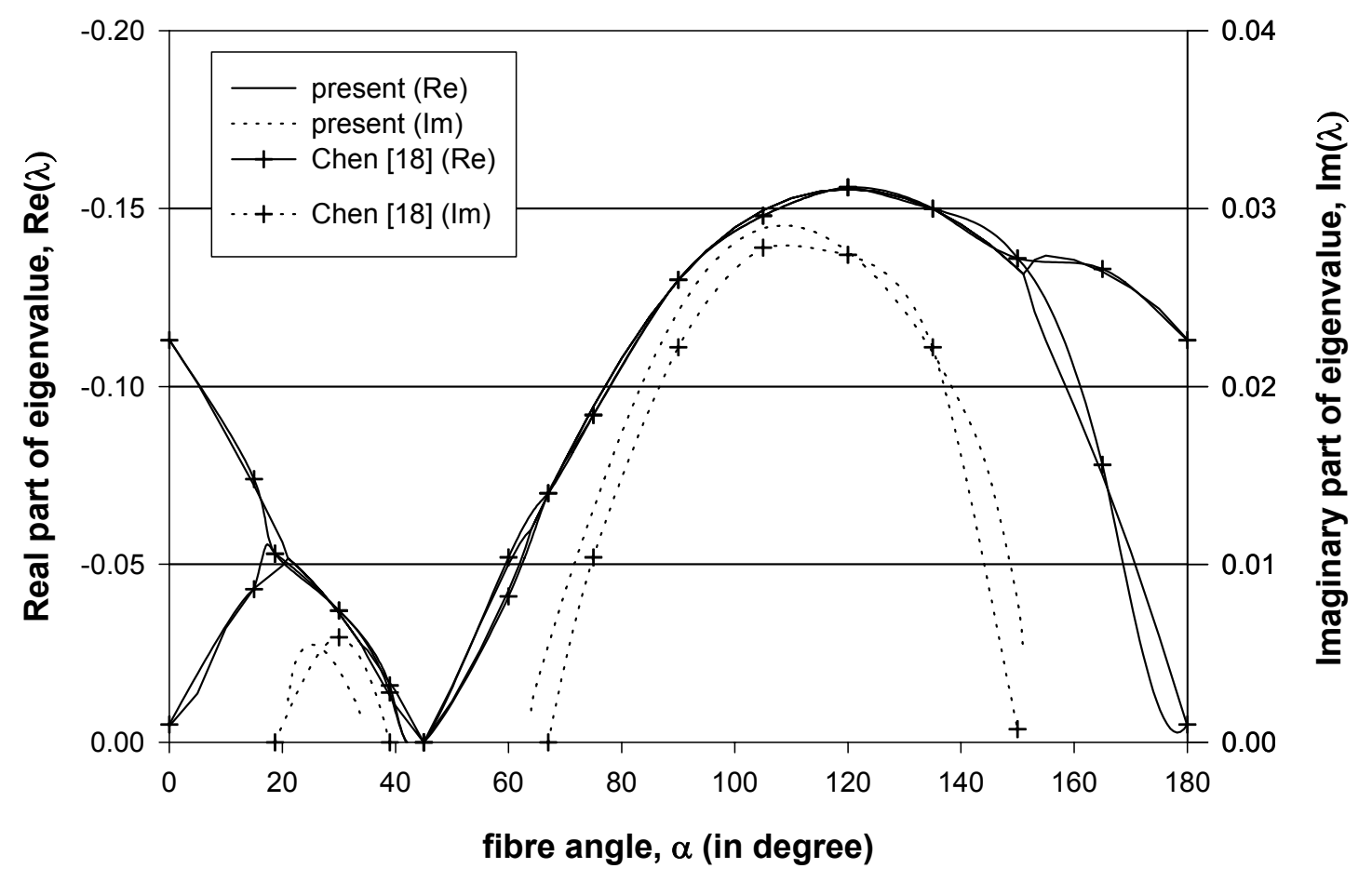

Fig.11. The real and imaginary parts of the computed eigenvalues for a 3/D bonded bimaterial junction with varying fibre orientation for material B, see Fig.1d 